\title{
PTEN deficiency reprogrammes human neural stem cells towards a glioblastoma stem cell-like phenotype
}

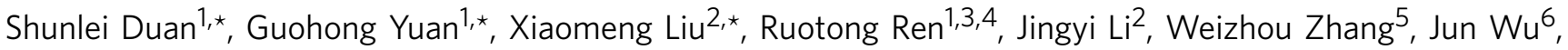
Xiuling $\mathrm{Xu}^{1}$, Lina Fu${ }^{1}$, Ying $\mathrm{Li}^{1}$, Jiping Yang${ }^{1}$, Weiqi Zhang ${ }^{1}$, Ruijun Bai ${ }^{3,4}$, Fei Yi ${ }^{7}$, Keiichiro Suzuki ${ }^{6,8}$, Hua Gao ${ }^{9}$, Concepcion Rodriguez Esteban ${ }^{6}$, Chuanbao Zhang ${ }^{10}$, Juan Carlos Izpisua Belmonte ${ }^{6}$, Zhiguo Chen ${ }^{11}$, Xiaomin Wang ${ }^{10}$, Tao Jiang ${ }^{10}$, Jing Qu ${ }^{4}$, Fuchou Tang ${ }^{2,12,13,14}$ \& Guang-Hui Liư 1,3,10,13

PTEN is a tumour suppressor frequently mutated in many types of cancers. Here we show that targeted disruption of PTEN leads to neoplastic transformation of human neural stem cells (NSCs), but not mesenchymal stem cells. PTEN-deficient NSCs display neoplasm-associated metabolic and gene expression profiles and generate intracranial tumours in immunodeficient mice. PTEN is localized to the nucleus in NSCs, binds to the PAX7 promoter through association with CAMP responsive element binding protein 1 (CREB)/CREB binding protein (CBP) and inhibits PAX7 transcription. PTEN deficiency leads to the upregulation of PAX7, which in turn promotes oncogenic transformation of NSCs and instates 'aggressiveness' in human glioblastoma stem cells. In a large clinical database, we find increased PAX7 levels in PTENdeficient glioblastoma. Furthermore, we identify that mitomycin $C$ selectively triggers apoptosis in NSCs with PTEN deficiency. Together, we uncover a potential mechanism of how PTEN safeguards NSCs, and establish a cellular platform to identify factors involved in NSC transformation, potentially permitting personalized treatment of glioblastoma.

\footnotetext{
${ }^{1}$ National Laboratory of Biomacromolecules, Institute of Biophysics, Chinese Academy of Sciences, Beijing 100101, China. ${ }^{2}$ Biodynamic Optical Imaging Center, College of Life Sciences, Peking University, Beijing 100871, China. ${ }^{3}$ FSU-CAS Innovation Institute, Foshan 528000, China. ${ }^{4}$ State Key Laboratory of Stem Cell and Reproductive Biology, Institute of Zoology, Chinese Academy of Sciences, Beijing 100101, China. ${ }^{5}$ Department of Pathology, Carver College of Medicine, University of lowa, lowa City, lowa 52242, USA. ${ }^{6}$ Gene Expression Laboratory, Salk Institute for Biological Studies, 10010 North Torrey Pines Road, La Jolla, California 92037, USA. ${ }^{7}$ Department of Molecular and Cellular Physiology, Stanford University School of Medicine, Stanford, California 94305, USA. 8 Universidad Católica San Antonio de Murcia (UCAM) Campus de los Jerónimos, No 135 Guadalupe, 30107 Murcia, Spain. ${ }^{9}$ Research Center for Translational Medicine, Shanghai East Hospital, School of Life Sciences and Technology, Tongji University, Shanghai 200092, China. ${ }^{10}$ Beijing Institute for Brain Disorders, Beijing 100069, China. ${ }^{11}$ Cell Therapy Center, Xuanwu Hospital Capital Medical University, Beijing 100053, China. ${ }^{12}$ Ministry of Education Key Laboratory of Cell Proliferation and Differentiation, Beijing 100871, China. ${ }^{13}$ Center for Molecular and Translational Medicine, CMTM, Beijing 100101, China. ${ }^{14}$ Peking-Tsinghua Center for Life Sciences, Peking University, Beijing 100871, China. ${ }^{\star}$ These authors contributed equally to this work. Correspondence and requests for materials should be addressed to J.Q. (email: qujing@ioz.ac.cn) or to F.T. (email: tangfuchou@pku.edu.cn) or to G.H.L. (email: ghliu@ibp.ac.cn).
} 
$\mathrm{P}$ hosphatase and tensin homolog (PTEN) is a potent tumour suppressor whose loss-of-function mutations are often encountered in human cancers. PTEN mutations are observed in $60 \%$ of glioblastoma multiforme (GBM) and are among the most frequent genetic alterations linked to $\mathrm{GBM}^{1}$. GBMs bearing PTEN loss-of-function mutations are usually associated with increased invasive behaviours and drug resistance $^{2-4}$. Glioblastoma stem cells (GSCs), the tumorigenic component of GBM, represent a rare cell population that are resistant to conventional radio- or chemo-therapy, and are presumably involved in cancer relapse $e^{5,6}$. Evidence from mouse tumour models reveals neural precursor/stem cells as the cell-oforigins for GBM or $\mathrm{GSCs}^{7-9}$, and GBM is postulated to be derived from transformed neural stem cells (NSCs) that undergo carcinogenic hits ${ }^{10}$. The high mutation rate of PTEN in GBM suggests its potential as one of the initiating oncogenic events or a key factor in promoting cancer aggressiveness, similarly as seen in endometrial cancer ${ }^{11}$. The correlation between PTEN deficiency and poor prognosis suggests a more complex role of PTEN loss in GBM progression. These observations raise an interesting question, that is, how PTEN loss leads to GBM initiation or promotes its progression?

Mouse models have been successfully used to investigate the roles of genetic mutations in triggering oncogenic NSC transformation and/or mediating GBM pathogenesis ${ }^{12,13}$. The known differences between mouse and human cancer biology, including differential telomere length, distinct utilization of p16 ${ }^{\text {INK4a }}-\mathrm{RB}$ versus p53 signalling and different sensitivity to anti-tumour drugs, however, have limited the degree to which insights derived from mouse models can be directly translated to human applications ${ }^{14-16}$. The advances in human stem cells and targeted gene editing technology have opened a new avenue for disease modelling and drug discovery ${ }^{17}$. Although many genetic disease models that are linked to development and ageing have been developed using human embryonic stem cells (ESCs) or induced pluripotent stem cells (iPSCs) ${ }^{17-24}$, very few human cancer models employing targeted genetic mutations in adult stem cells have been established for gaining mechanistic insights or testing drug efficacies ${ }^{25,26}$.

Considering the potential of NSCs being the cell-of-origin for human GBM, and PTEN deletion has frequently been reported in GBM, we hypothesize that PTEN functions as a gatekeeper to protect human NSCs from neoplastic transformation. Accordingly, we generated PTEN-deficient human NSCs by targeted gene editing. PTEN deficiency resulted in a reprogramming of NSCs towards a GSC-like phenotype in a highly lineage-specific mechanism primarily through transcriptional activation of $P A X 7$, a pathway that is also linked to the aggressive characteristics of GSCs in GBM patients.

\section{Results}

PTEN deficiency leads to neoplastic features in NSCs. To generate isogenic PTEN-null human NSC lines, we first utilized transcription activator-like effector nuclease (TALEN)-mediated homologous recombination (HR) to delete the exon 1 of PTEN gene in human ESCs (Fig. 1a). Successful gene targeting at PTEN locus was verified by genomic PCR (Fig. 1b). Immunofluorescence staining revealed a punctate staining pattern of PTEN in the nucleus of wild-type (WT) ESCs, which was absent in PTEN homozygous knockout $\left(P_{T E N}^{-l-}\right)$ ESCs (Fig. 1c). Western blot further confirmed the loss of PTEN protein in $P T E N^{-/-}$ESCs (Fig. 1d). The deficiency of PTEN, however, did not alter ESC-associated features such as morphology, DNA hypo-methylation at OCT4 promoter and the expression of pluripotency markers OCT4, SOX2, NANOG and
TRA-1-81 (Supplementary Fig. 1a-c). PTEN ${ }^{-1-}$ ESCs also maintained the capability of differentiating into endoderm, mesoderm and ectoderm lineages in vivo, as did WT ESCs (Supplementary Fig. 1d).

To study the role of PTEN in NSCs, we derived NSCs from both WT and PTEN ${ }^{-1-}$ ESCs. We confirmed the loss of PTEN in $P T_{E N}{ }^{-1-}$ NSCs (Fig. 1e,f and Supplementary Fig. 2a). Both the WT and $P T E N^{-I-}$ NSCs exhibited typical features of neural progenitors, including the expression of neural progenitor markers PAX6, SOX2 and Nestin, the absence of pluripotency-related antigen TRA-1-81, the hyper-methylation of OCT4 promoter, as well as the enrichment of H3K4me3 levels at PAX6 and Nestin loci (Fig. 1g and Supplementary Fig. 2b-f). More importantly both WT and $P T E N^{-1-}$ NSCs were able to efficiently differentiate into neurons and astrocytes in vitro (Fig. $1 \mathrm{~g}$ and Supplementary Fig. 2g), confirming their NSC identity.

Next, we investigated whether PTEN-deficient NSCs could have acquired neoplastic potentials. $P T E N^{-/}$NSCs exhibited accelerated growth under suspended neurosphere culturing condition (Supplementary Fig. 3a), accompanied by a mild increase in the population of cells retained in the $S$ and G2/M phases and a concomitant decrease in the population of cells in the G0/G1 phases (Supplementary Fig. 3b). Consistent with an acquired growth advantage, $P T E N^{-1}-$ NSCs exhibited enlarged nuclei size, increased number of nucleoli and elevated rRNA transcripts (Supplementary Fig. 3c-e). It has been reported that PTEN-loss is associated with genomic instability in mouse embryonic fibroblasts (MEFs) ${ }^{27}$. We performed karyotyping and genome-wide copy number variation (CNV) analyses by deep sequencing and did not observe any discernible chromosomal aberrations in $P T E N^{-1-}$ NSCs compared with the WT NSCs (Supplementary Fig. 3f, g). In agreement with a neoplastic potential, $P T E N^{-/-}$NSCs showed resistance to stresses-induced cell death (Supplementary Fig. 3h,i) ${ }^{28,29}$, as well as significant elevation in cell migration and clonal expansion abilities (Fig. 2a,b and Supplementary Fig. 4a,b). Re-introduction of PTEN into the PTEN ${ }^{-/-}$NSCs partially rescued these neoplastic phenotypes (Fig. 2c,d and Supplementary Fig. 4c,d). In addition, knockdown of PTEN using small hairpin (sh) RNA in either ESC- or iPSC-derived NSCs recapitulated the aggressive phenotypes observed in $P T E N^{-1-}$ NSCs (Supplementary Figs $4 \mathrm{e}, \mathrm{f}$ and $5 \mathrm{a}-\mathrm{e})$. Altogether, these in vitro experiments demonstrated that PTEN deficiency endowed NSCs with neoplastic potential.

Next we explored whether PTEN deficiency is linked to tumour initiation in NSCs using an in vivo mouse model. We implanted WT or PTEN-deficient NSCs expressing luciferase into the brains of immunocompromised NOD/SCID mice. PTEN-deficient NSCs were able to grow efficiently and form intracranial tumours in vivo as evidenced by both positive luminescence and magnetic resonance imaging (MRI) signals (Fig. 2e and Supplementary Fig. $6 \mathrm{a}-\mathrm{c}$ ). Similar to primary human $\mathrm{NSCs}^{30}$, WT ESC-derived NSCs did not expand in the brain (Fig. 2e). Haematoxylin and eosin (H\&E) staining of brain slices showed a substantial expansion of the grafted PTEN $-/-$ NSCs, with characteristics of neoplastic cells intruding adjacent normal brain tissue and histological resemblance of intracranial tumour derived from the grafted human GSCs (Fig. 2f and Supplementary Fig. 6e) ${ }^{30-33}$. Occasionally we observed invasion of neoplastic cells into other brain regions, intra-tumour vasculatures and non-classic necrosis in the mouse brain implanted with $P T E N^{-/}$NSCs for a longer course ( $\sim 70$ days; Supplementary Fig. 6e). PTEN ${ }^{-1-}$ NSCderived neoplasm also expressed typical mixed markers of GBM including Nestin, SRY box 2 (SOX2), glial fibrillary acidic protein (GFAP), neuron-specific beta -III tubulin (Tuj1)

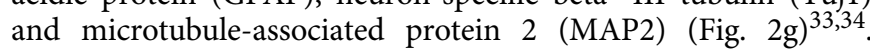


a

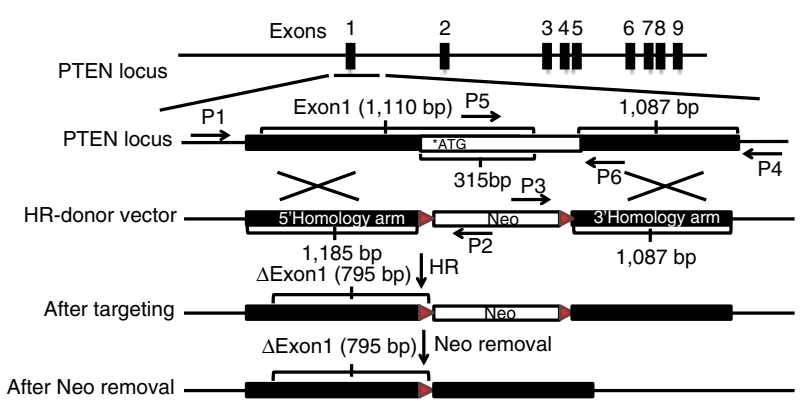

b

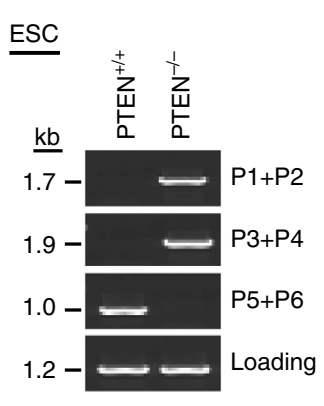

C

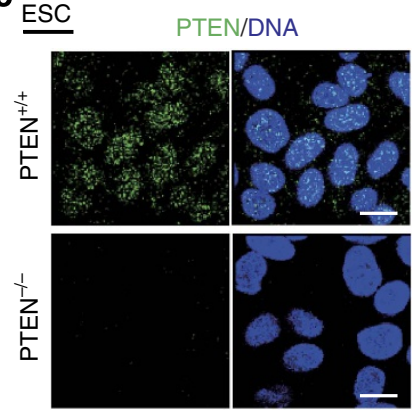

d

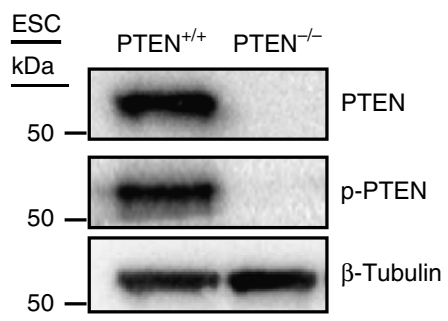

e NSC

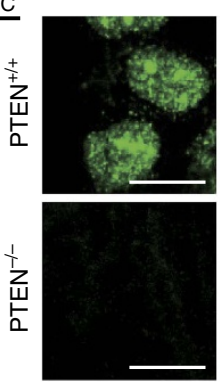

PTEN/DNA

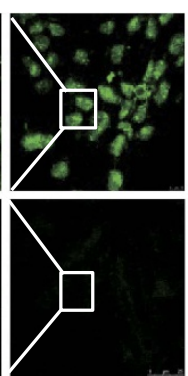

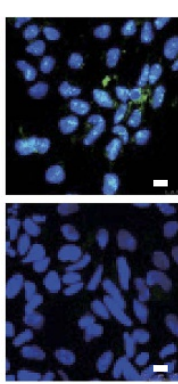

f

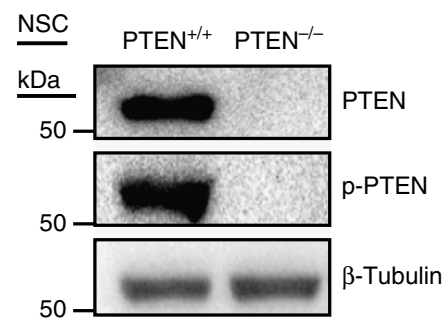

9

NSC

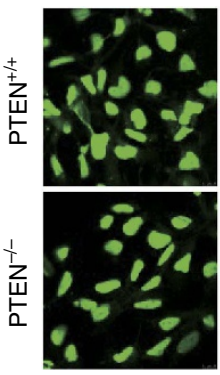

SOX2/PAX6/DNA

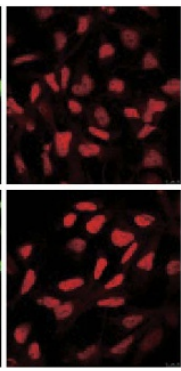

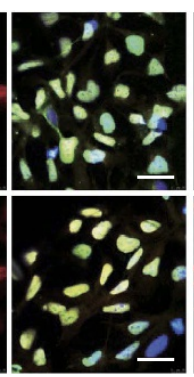

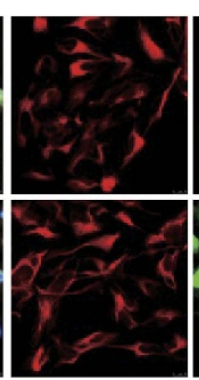

Nestin/Ki67/DNA
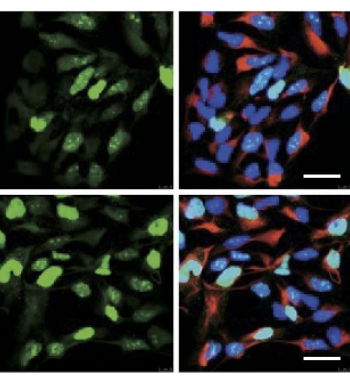

Neuron

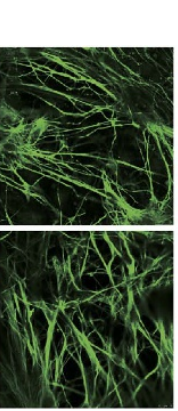

Tuj1/MAP2/DNA

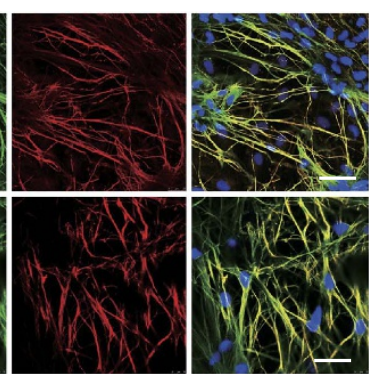

Figure 1 | Generation and characterization of PTEN-deficient NSCs. (a) Schematic representation of TALEN-based PTEN targeting strategy. Primers used for $\mathbf{b}$ are shown as arrows (P1-P6). The donor vector includes a neomycin-resistance cassette (Neo) allowing for positive selection. (b) PCR analysis of WT and PTEN - - ESCs using primer pairs indicated (P1 + P2: 1,744 bp; P3 + P4: 1,872 bp; P5 + P6: 1,035 bp). (c) Immunofluorescence analysis performed on WT and PTEN ${ }^{-1-}$ ESCs with an anti-PTEN antibody. PTEN was absent in the PTEN ${ }^{-1-}$ ESCs. Nuclei were stained with Hoechst 33342. Scale bars, $12.5 \mu \mathrm{m}$. (d) Immunoblotting verified the absence of PTEN protein in PTEN ${ }^{-/-}$ESCs with anti-PTEN and anti-phospho-PTEN (Ser380) antibodies. $\beta$-Tubulin was used as loading control. (e) Immunostaining of PTEN in WT and PTEN ${ }^{-/-}$NSCs with an anti-PTEN antibody. Scale bars, $10 \mu \mathrm{m}$. (f) Immunoblotting analysis of PTEN and phospho-PTEN in WT and PTEN $-/$ - NSCs. (g) Immunostaining of neural progenitor- (left) and neuron- (right) specific markers in WT and PTEN ${ }^{-1-}$ NSCs (left) and their neuronal derivatives (right). Scale bars, $25 \mu \mathrm{m}$ (NSC) and $50 \mu \mathrm{m}$ (neuron).

In agreement with several studies using $\mathrm{GSCs}^{35,36}$, the neoplastic phenotype in $P T E N^{-1-}$ NSCs was mediated by their CD133-positive, rather than CD133-negative population (Supplementary Fig. 6f). The in vivo aggressiveness of $P T E N^{-1-}$ NSCs was able to be suppressed by expressing exogenous PTEN (Supplementary Fig. 6d), suggesting a direct involvement of PTEN in suppressing the neoplastic phenotype in NSCs.

A full-fledged cancer phenotype normally needs a combination of multiple oncogenic mutations. To test whether the neoplastic phenotype in PTEN-deficient NSCs can be aggravated by inactivation of another tumour suppressor, we knocked down one of the most well-known tumour suppressor p53 in PTEN-deficient NSCs ${ }^{37}$. As expected, we observed dramatic neoplastic transformation in NSCs when p53 and PTEN were simultaneously inactivated (Supplementary Fig. 7a-e). Next, we examined whether the intracranial niche of the brain was required for tumorigenesis mediated by PTEN deletion in
NSCs. When $P T E N^{-1-}$ NSCs were implanted into NOD/SCID mice via the subcutaneous route, we did not detect any neoplasmlike growth, suggesting that $P T E N^{-1-}$ NSCs required a supportive brain environment for tumour initiation.

We also investigated whether PTEN deletion had impact on neoplastic transformation in other human adult stem cells. To this end, we differentiated $P T E N^{-1}-$ ESCs to mesenchymal stem cells (MSCs) ${ }^{22,23}$. The $P T E N^{-1-}$ MSCs expressed specific markers, such as CD73, CD90, CD105, and were absent of MSC-irrelevant markers, including CD34, CD43 and CD45 (Supplementary Fig. 8a,b). The $P T E N^{-}-$MSCs exhibited $^{-}$ typical features of premature senescence, including the positive SA- $\beta$-Gal staining, expression of senescence-related gene transcripts and the compromised migration and clonal expansion (Supplementary Fig. 8c-g). Likewise, knockdown of PTEN using shRNA led to senescence in WT human MSCs (Supplementary Fig. 8h,i). Similar accelerated ageing phenotypes were also observed in the fibroblast-like cells 

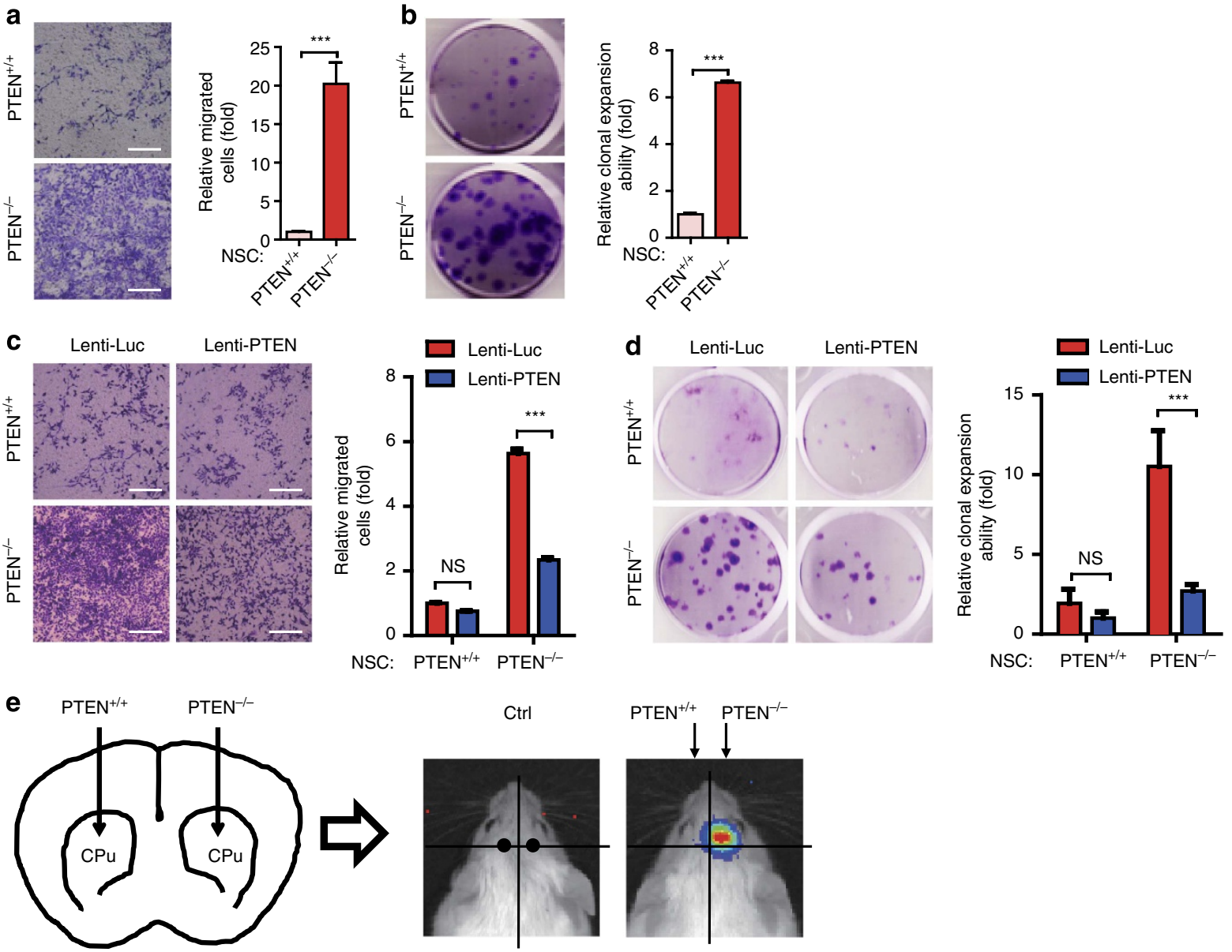

f
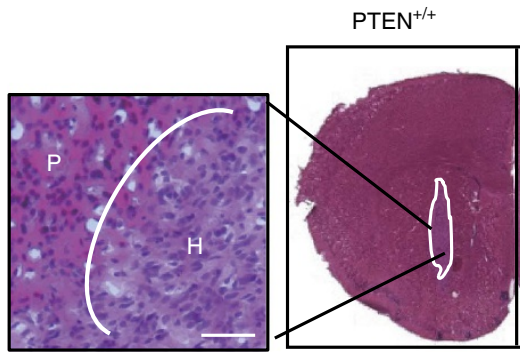

PTEN ${ }^{-/}$

g

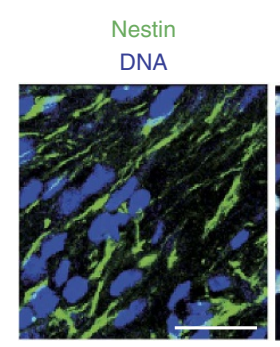

sox2

Human nucle

DNA

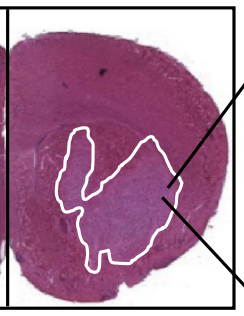

GFAP

Human nucle

DNA
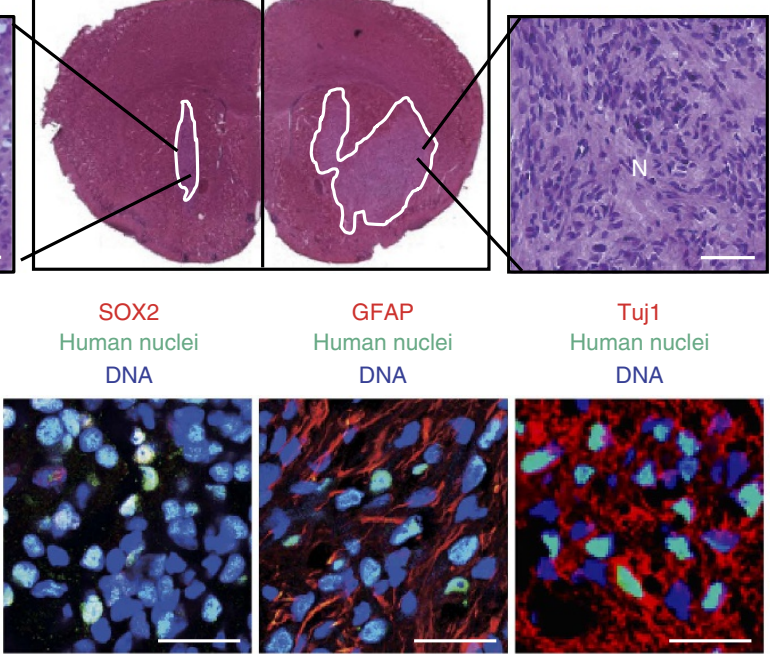

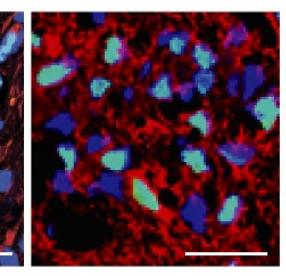

\section{Tuj1}

Human nuclei

DNA
MAP2

Human nuclei

DNA

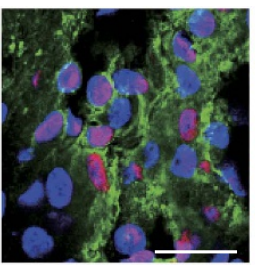

Nestin

GFAP

DNA

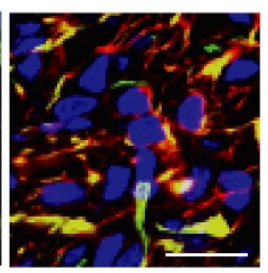

Figure 2 | PTEN-deficient NSCs demonstrated neoplastic features in vitro and in vivo. (a) Migration abilities of WT and PTEN ${ }^{-/-}$NSCs were evaluated by Transwell assays. Relative cell migration efficiency was determined. Data are shown as mean \pm s.e.m. $n=3$. ${ }^{\star \star \star} P<0.001$ ( $t$-test). Scale bars, 1 mm. (b) Clonal expansion analysis in WT and PTEN ${ }^{-1-}$ NSCs. Crystal violet staining-positive cells were calculated and presented as fold induction using Image J. Data are shown as mean \pm s.e.m. $n=4 .{ }^{\star \star \star} P<0.001$ ( $t$-test). (c) Cell migration analyses of NSCs transduced with a PTEN expression vector (Lenti-PTEN) or a control vector (Lenti-Luc). Data are shown as mean \pm s.e.m. $n=6 .{ }^{\star \star \star} P<0.001$ ( $t$-test); NS, not significant. Scale bars, $1 \mathrm{~mm}$. (d) Clonal expansion analysis of NSCs transduced with Lenti-PTEN or Lenti-Luc. Data are shown as mean \pm s.e.m. $n=3$. ${ }^{\star \star \star} P<0.001$ ( $t$-test); NS, not significant. (e) Representative photon flux images from the brain of NOD/SCID mice implanted with WT (left) and PTEN ${ }^{-/}$- (right) NSCs expressing luciferase (also see Supplementary Fig.6a for details). Images were taken $20 \mathrm{~min}$ after L-luciferin was injected intraperitoneally (i.p.). $n=3$. (f) H\&E staining and photomicrograph of a section of entire brain showing dramatic neoplastic expansion of PTEN ${ }^{-/}-\mathrm{NSC}$ relative to WT control ( $2 \times 10^{6}$ cells per injection), 35 days after cells were injected into the contralateral corpus striatum. $\mathrm{H}$ represents residual human NSC graft without expansion, N represents neoplasm and $P$ represents mouse brain parenchyma. Scale bars, $500 \mu \mathrm{m}$. $(\mathbf{g})$ Immunostaining of brain sections described in $\mathbf{f}$ with antibodies against the indicated antigens. Human nuclei, human nucleus antigen. DNA was stained with Hoechst 33342. Scale bars, $25 \mu \mathrm{m}$. 
derived from in vivo differentiated $P T E N^{-/}$ESCs (Supplementary Fig. 8j). The pro-senescence effect observed in MSCs appears to be specific to PTEN deficiency, as knockdown of p53 in MSCs led to strengthened proliferation and migration instead (Supplementary Figs $7 \mathrm{e}$ and $8 \mathrm{k}, \mathrm{l}$ ). Herein, these observations indicate that PTEN deficiency preferentially b

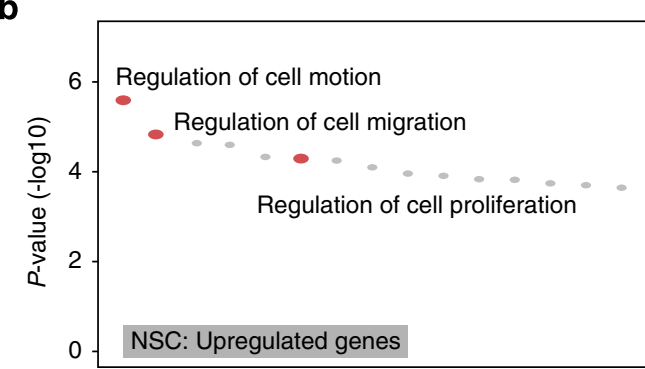

Ranking of GO_biological process

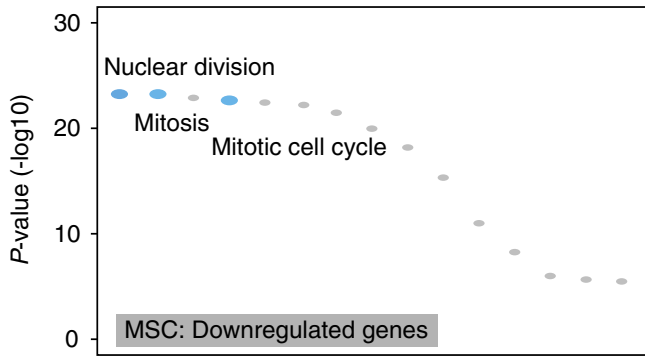

Ranking of GO_biological process
C
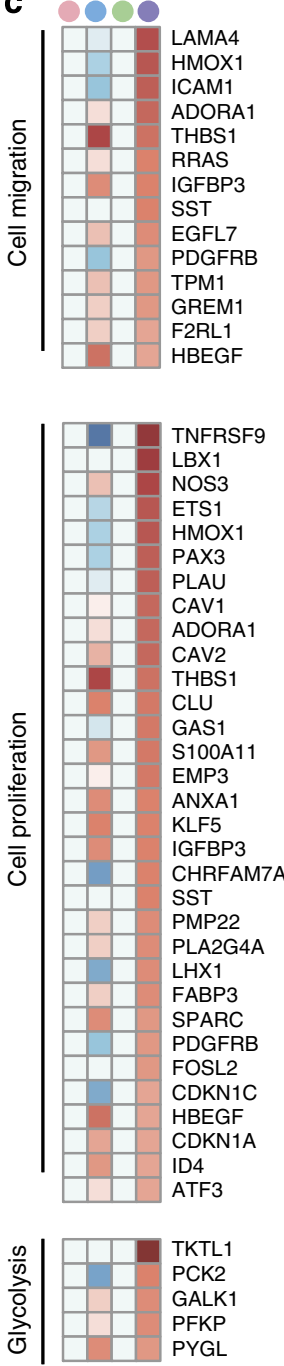

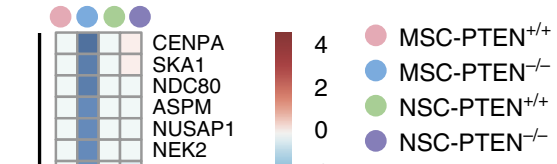

d Upregulated genes
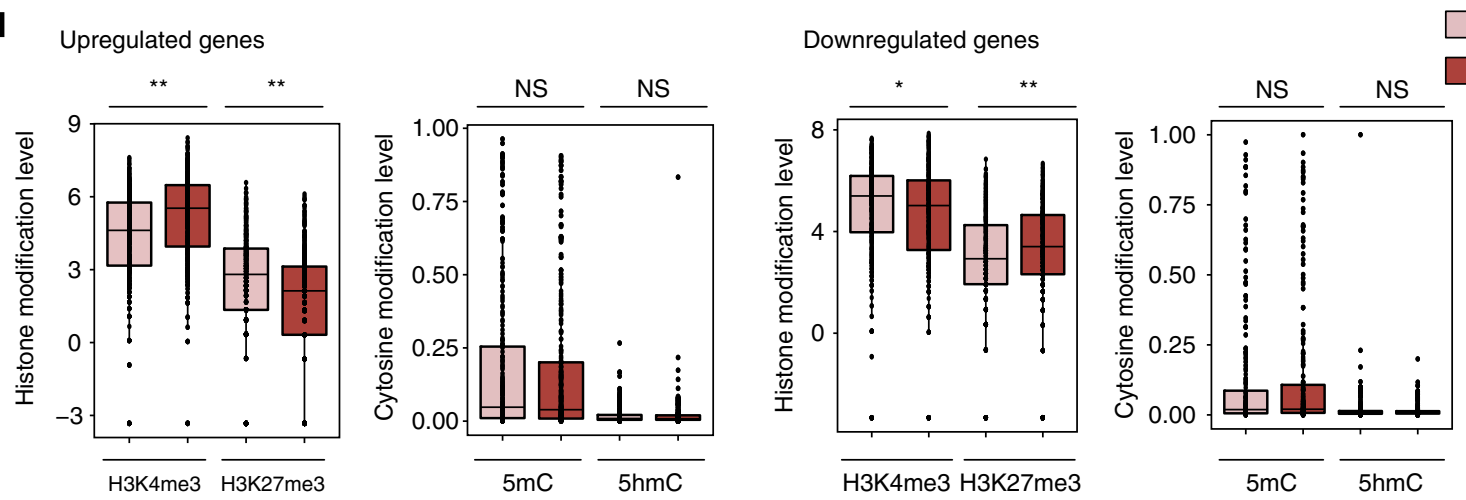

NSC PTEN ${ }^{+/+}$ NSC PTEN ${ }^{-/}$

Figure 3 | Transcriptome and epigenome analyses in PTEN-deficient stem cells. (a) Glycolysis flux analysis measured by extracellular acidification rates

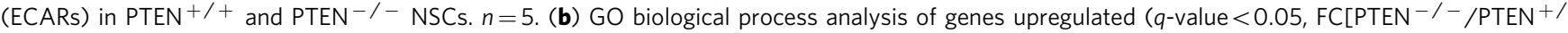

$\left.{ }^{+}\right]>2$ ) after PTEN knockout in NSCs and genes downregulated ( $q$-value $<0.05$, FC $[$ PTEN $-/-/$ PTEN $\left.+/+]<0.5\right)$ after PTEN knockout in MSCs. (c) Heat map displaying the upregulated genes ( $q$-value $\left.<0.05, \mathrm{FC}_{\mathrm{PTEN}}{ }^{-/-} / \mathrm{PTEN}{ }^{+/+}\right]>2$ ) in PTEN-deficient NSCs (left) and the downregulated genes ( $q$-value $<0.05$, FC $\left._{\text {PTEN }}-/-/ \mathrm{PTEN}^{+/+}\right]<0.5$ ) in PTEN-deficient MSCs (right). The representative enriched GO terms of biological processes were shown at the left side of each panel. (d) Boxplots showing H3K4me3, H3K27me3, 5mC and $5 \mathrm{hmC}$ levels in the region of $1 \mathrm{~kb}$ upstream and $1 \mathrm{~kb}$ downstream of TSS of differentially expressed genes between PTEN ${ }^{-/-}$and WT NSCs including the upregulated genes (left panels, $q$-value $<0.05$,

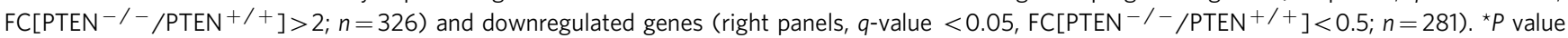
$<0.05$ (Wilcoxon signed-rank test); ${ }^{\star \star} P$ value $<0.01$ (Wilcoxon signed-rank test); NS, not significant. 
induces neoplastic transformation in NSCs by promoting proliferation and invasiveness; however, in the case of mesodermal cell types such as MSCs and fibroblasts, PTEN deletion primarily triggers cellular senescence.

PTEN $^{-/-}$NSCs display oncogenic gene expression changes. As PTEN is a suppressor of PI3K-AKT pathway primarily via its activity as a lipid phosphatase ${ }^{38,39}$, we next examined the downstream signalling pathways that are regulated by PTEN. $P_{T E N^{-1-}}$ NSCs exhibited an activated AKT pathway as evidenced by the phosphorylation of AKT and its downstream targets such as mammalian target of rapamycin (mTOR) and glycogen synthase kinase 3 beta (GSK3 $\beta$ ) when compared with the WT NSCs (Supplementary Fig. 9a,b). In contrast, AKT pathway activities were not affected by PTEN status in MSCs (Supplementary Fig. 9a), suggesting a different regulatory mechanism for AKT activation in MSCs.

Given that the AKT and mTOR activations are important for metabolic regulation of cancer cells, we further investigated the metabolic changes conferred by PTEN deficiency in NSCs. Mass spectrometry-based screening identified 16 metabolites were significantly upregulated in PTEN ${ }^{-/-}$NSCs compared with WT NSCs (Supplementary Fig. 9c,d and Supplementary Data 1). Among the 16 metabolites some belong to glycolytic pathway including glucose, glucose-6-phosphate and fructose-6-phosphate. Seahorse analysis indicated an increased glycolysis activity in $P_{T E N^{-1}}$ NSCs (Fig. 3a and Supplementary Fig. 9e). Consistently, $P T E N^{-1-}$ NSCs produced threefold more lactate acid content than that produced by WT NSCs (Supplementary Fig. 9f), and the ratio between ADP or AMP and ATP were increased in $P T E N^{-1-}$ NSCs relative to that of WT NSCs (Supplementary Fig. 9c and Supplementary Data 1). These data strongly support that $P T E N^{-/}$NSCs have dysregulated glucose metabolism and favour aerobic glycolysis, a feature characteristic of GBM and most cancer cells ${ }^{40,41}$. The PTEN-deficiencyinduced metabolic change was specific to NSCs, because these metabolites did not increase in $P T E N^{-1}$ ESCs when compared with WT ESCs (Supplementary Figs 9d,f and Supplementary Data 1).

Next, we investigated the impact of PTEN loss on global transcriptomic landscape of NSCs. We performed RNA-seq analyses for both WT and PTEN ${ }^{-1-}$ NSCs as well as MSCs. Using a threshold of twofold change with $q$-values less than 0.05 , we identified 607 genes differentially expressed between WT and $P_{\text {TEN }}^{-1-}$ NSCs, among which 326 were upregulated (Supplementary Fig. 10a-e and Supplementary Data 2). Gene ontology (GO) terms enriched in upregulated genes are linked to GSC-related functions (Fig. $3 \mathrm{~b}$ and Supplementary Data 2).
Notably, most of these genes belong to signalling pathways important for cell motion, migration and proliferation (Fig. 3b,c, Supplementary Fig. 10f,g and Supplementary Data 2). Consistent with the metabolic changes, a number of enzymes involved in glycolysis were upregulated in $P T E N^{-1-}$ NSCs (Fig. 3c and Supplementary Fig. 10f,g). These gene expression changes were partially rescued by re-introduction of PTEN in $P T E N^{-P-}$ NSCs (Supplementary Fig. 10h). In contrast, PTEN deletion in MSCs resulted in differential expression of 1,854 genes (Supplementary Fig. 10a-e). Consistent with the non-neoplastic phenotype in PTEN ${ }^{-1}-$ MSCs, PTEN deficiency led to a coordinated downregulation of genes required for cell cycle process (Fig. 3b,c and Supplementary Data 2), which is in agreement with a molecular signature of accelerated senescent MSCs ${ }^{23}$. Of note is that only few gene expression changes caused by PTEN deficiency overlap between NSCs and MSCs (Supplementary Fig. 10d and Supplementary Data 2), serving a possible explanation for the dramatically different cellular phenotypes observed between PTEN-deficient NSCs and MSCs.

To explore whether global transcriptome change in $P T E N^{-1-}$ NSCs are linked to epigenetic alterations, we performed chromatin immunoprecipitation sequencing (ChIP-seq) to map genome-wide distribution of $\mathrm{H} 3 \mathrm{~K} 4 \mathrm{me} 3, \mathrm{H} 3 \mathrm{~K} 27 \mathrm{me} 3$, as well as reduced representation bisulfite sequencing (RRBS) and Tet-assisted RRBS (TA-RRBS) for 5-methylcytosine (5mC) and 5-hydroxymethylcytosine $(5 \mathrm{hmC})$ in WT or $P T E N^{-/-}$NSCs (Fig. 3d and Supplementary Fig. 11a,b). Notably, the upregulated genes showed significant increase of $\mathrm{H} 3 \mathrm{~K} 4 \mathrm{me} 3$ and decrease of $\mathrm{H} 3 \mathrm{~K} 27 \mathrm{me}$, whereas the downregulated genes were associated with diminished $\mathrm{H} 3 \mathrm{~K} 4 \mathrm{me} 3$ and elevated $\mathrm{H} 3 \mathrm{~K} 27 \mathrm{me} 3$, at their promoter regions (Fig. 3d). We did not observe any global associations between $5 \mathrm{mC}$ (or $5 \mathrm{hmC}$ ) and gene expression changes (Fig. 3d). These observations suggested that changes in histone modifications including $\mathrm{H} 3 \mathrm{~K} 4 \mathrm{me} 3$ and $\mathrm{H} 3 \mathrm{~K} 27 \mathrm{me} 3$ could account for PTEN-loss-induced transcriptional dysregulation in PTEN $^{-1-}$ NSCs.

PAX7 mediates oncogenic features in PTEN ${ }^{-1}-$ NSCs. Among the upregulated genes, transcription factor $P A X 7$ was at the top of the list and expressed 155-fold higher in PTEN ${ }^{-1}-$ NSCs than in WT NSCs (Supplementary Data 2). Also it is known that elevated levels of PAX7 in embryonal rhabdomyosarcoma cells are associated with increased migration and invasiveness ${ }^{42,43}$. Thus, we focused on a potential role of PAX7 in regulating neoplastic transformation of NSCs.

We confirmed the elevated PAX7 expression in $P T E N^{-1-}$ NSCs relative to WT NSCs, using reverse transcriptionquantitative PCR (RT-qPCR) and western blotting (Fig. 4a and

Figure 4 | Transcriptional activation of PAX7 underlies oncogenic features in PTEN-deficient NSCs. (a) RT-qPCR analyses of PAX7 expression in WT and PTEN ${ }^{-/-}$NSCs. $n=3$. ${ }^{* \star \star} P<0.001$ ( $t$-test). (b) RT-qPCR showing reduced transcripts of PAX7 in PTEN ${ }^{-/}-$NSCs transduced with lentivirus encoding PTEN. $n=3$. ${ }^{\star \star \star} P<0.001$ (t-test). (c) Cell migration analysis in WT and PTEN ${ }^{-1}-$ NSCs transduced with control shRNA or PAX7 shRNA. $n=5$. ${ }^{\star} P<0.05$ (t-test); and ${ }^{\star \star \star} P<0.001$ (t-test). (d) MRI analysis of intracranially implanted WT and $P T E N^{-/}-$NSCs pre-transduced with control or PAX7 shRNA. Relative volumes of tumours are presented. $n=4$. ${ }^{\star \star} P<0.01$ ( $t$-test), and ${ }^{\star \star \star} P<0.001$ ( $t$-test). (e) Cell migration analysis in PTEN ${ }^{-1}-$ NSCs transduced with the lentiviral vector encoding dominant negative mutants of PAX7 (DN1: a.a.1-263; DN2: a.a.1-318) or a luciferase control (Luc). $n=3$. ${ }^{\star \star \star} P<0.001$ ( $t$-test). (f) Cell migration analysis in WT NSCs transduced with the lentiviral vector encoding PAX7. $n=3$. ${ }^{\star \star \star} P<0.001$ ( $t$-test). (g) ChIP-PCR showing the association of endogenous PTEN with PAX7 promoter in WT NSCs. The PAX7 promoter was amplified by PCR from either genomic DNA as input (lanes 1 and 4 ) or antiPTEN immunoprecipitated DNA (lanes 3 and 6). (h) Luciferase reporter assay showed that the PTEN-docking DNA element described in $\mathbf{g}$ has a higher cisactivation ability when PTEN was depleted in NSCs. $n=3$. ${ }^{\star \star} P<0.01$ ( $t$-test). (i) Immunoblotting analysis of CREB and phospho-CREB (Ser133) expression in WT and PTEN ${ }^{-/-}$NSCs. $\beta$-Actin was used as loading control. (j) Upper panel: Identification of CRE-containing genes in the human genome by the FIMO software tool. Lower panel: Bioinformatic analysis predicated that 167 (51.2\%) of 326 genes upregulated in PTEN ${ }^{-/}-$NSCs are potential CREB-targets. (k) ChIP-qPCR analysis for PTEN, p-CREB and CBP enrichment within the PTEN-docking DNA region at the PAX7 promoter in WT and PTEN ${ }^{-/-}$NSCs. P1-P8 indicate the different sub-regions. ${ }^{\star \star \star} P<0.001$ ( $t$-test); NS, not significant. (I) Cell migration analysis (top panels) of $P$ TEN ${ }^{-/-}$NSCs transduced with the lentiviral vector encoding PTEN, PTEN(G129E), PTEN(Y138L) or a luciferase control (Luc), and the expression of PTEN was determined by immunofluorescence (lower panels). Scale bars, $1 \mathrm{~mm}$ (migration assay) and $50 \mu \mathrm{m}$ (IF). $n=3$. ${ }^{\star \star \star} P<0.001$ ( $t$-test). 
Supplementary Fig. 12a). In contrast, the expression of PAX7 in $P_{\text {TEN }}^{-1-}$ MSCs did not increase when compared with WT MSCs (Supplementary Fig. 12b). The upregulation of PAX7 in
PTEN $^{-1-}$ NSCs was directly caused by the absence of PTEN as reintroduction of PTEN in these cells repressed PAX7 expression (Fig. 4b and Supplementary Fig. 12c). a

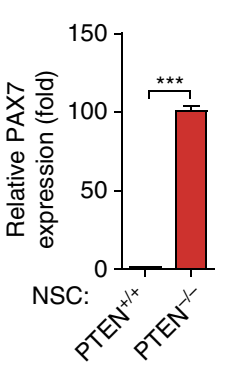

e

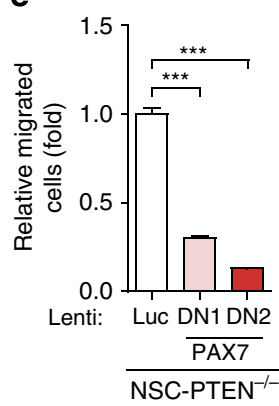

j

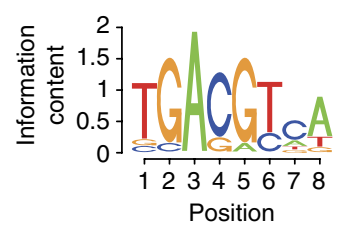

326

FPKM $\left(\right.$ PTEN $^{-1-}$ vs PTEN $\left.{ }^{+/ 4}\right)>2$,

$P$ value $<0.05, q$ value $<0.05$

167

With CREB-binding site

$P<1 \mathrm{e}-4$

108

H3K4me3 ratio

$\left(\right.$ PTEN $^{-1-}$ vs PTEN $\left.^{+/+}\right)>2$

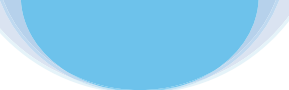

b

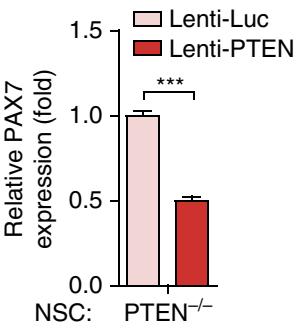

f

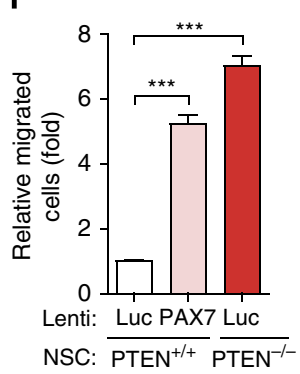

C

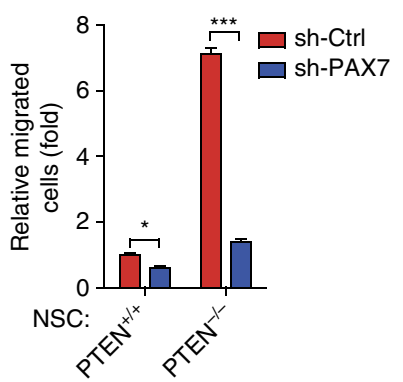

g

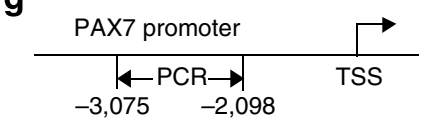

IP: IgG $\frac{\text { PTEN }^{+/+}}{-+-} \frac{\text { PTEN }^{-/-}}{-+-} \mathrm{M}$

IP: $\alpha$ PTEN

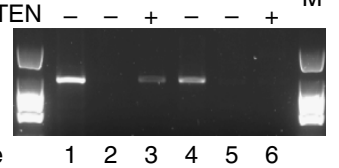

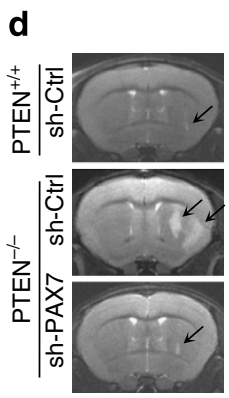

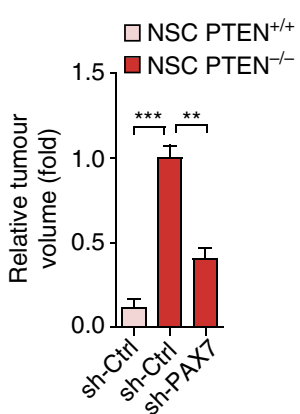

h

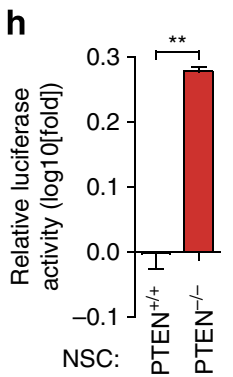

i

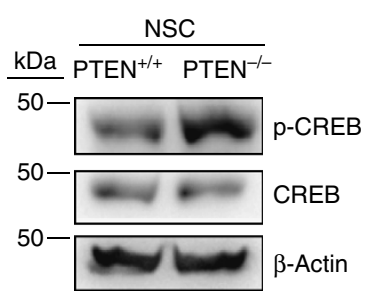

k

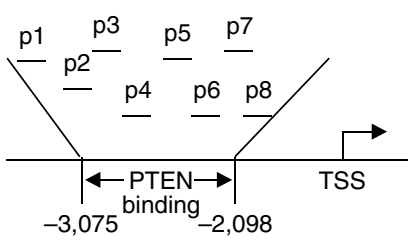

口 $\mathrm{PTEN}^{+/+}$IgG

口 PTEN $-1-\operatorname{lgG}$

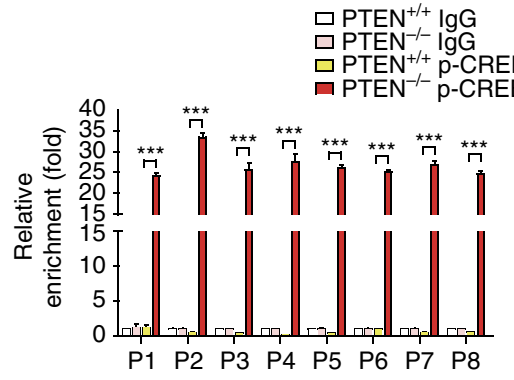

I

$\mathrm{PTEN}^{-/-}$
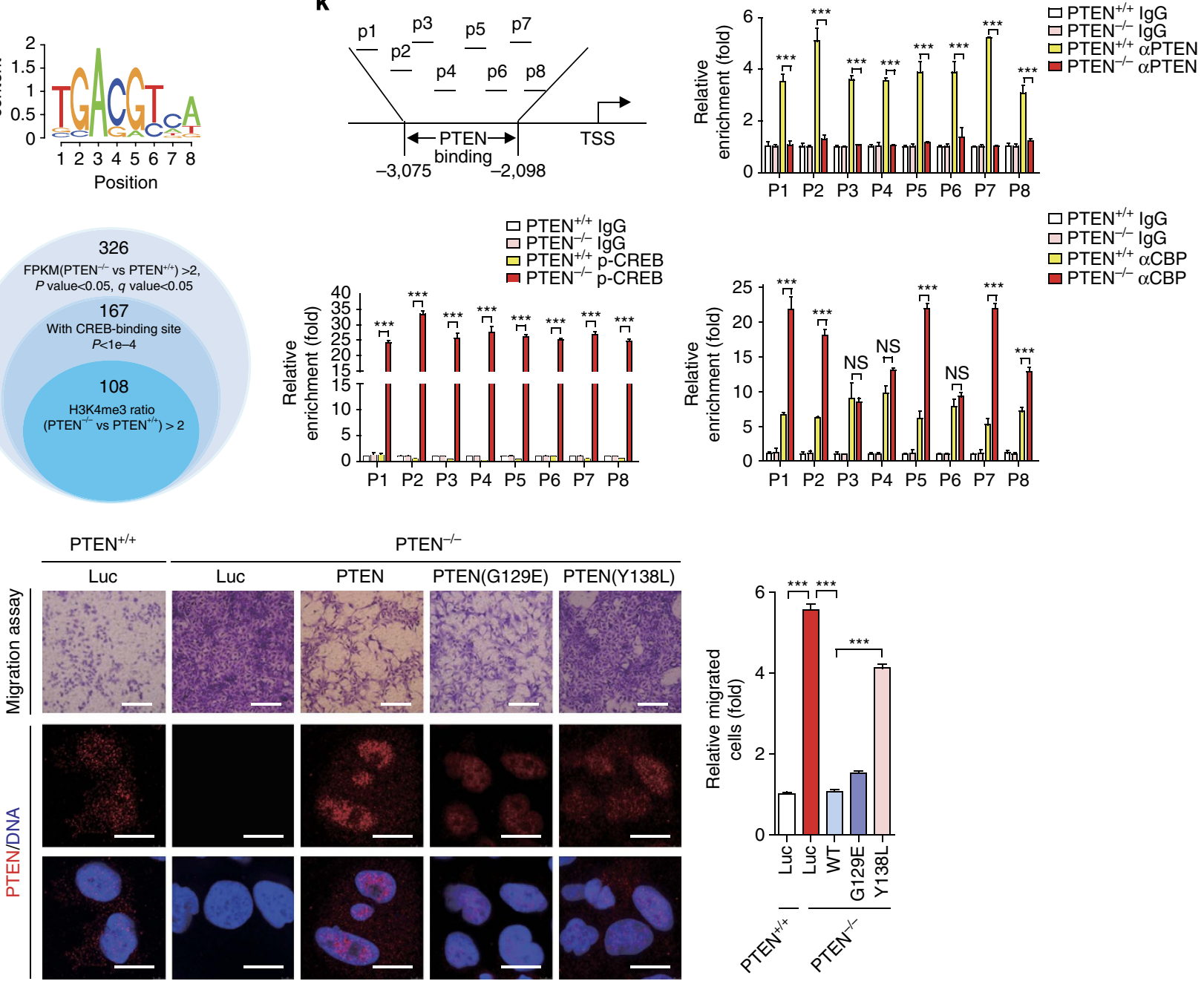

$\begin{array}{llllllll}\text { P1 } & \text { P2 } & \text { P3 } & \text { P4 } & \text { P5 } & \text { P6 } & \text { P7 } & \text { P8 }\end{array}$
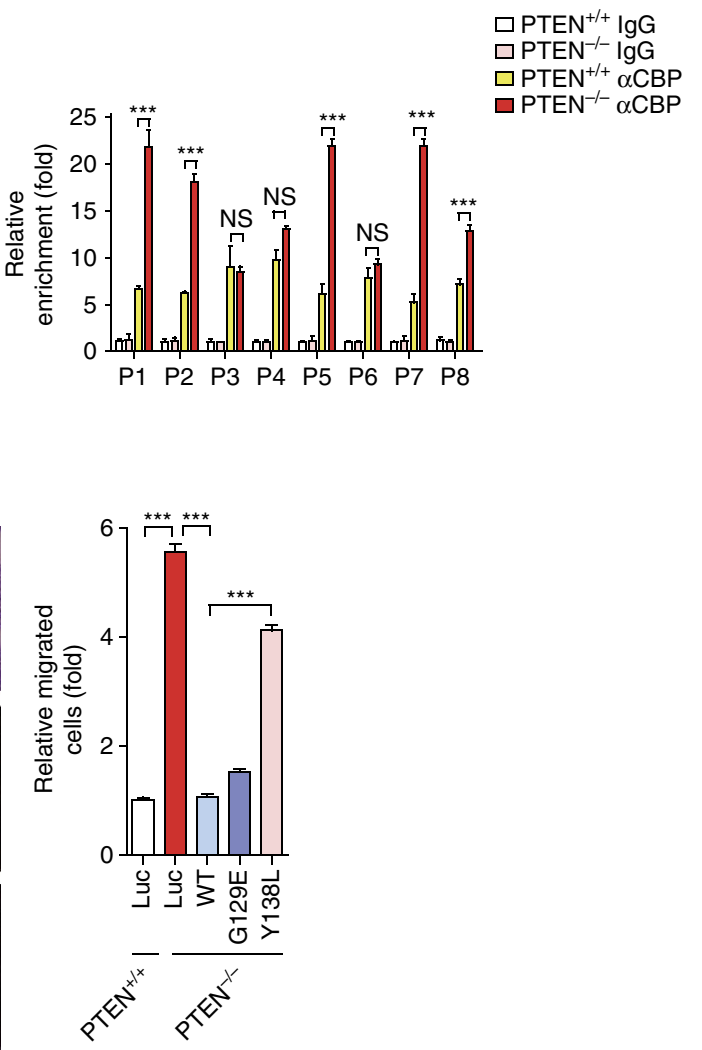
To investigate whether PAX7 mediates the neoplastic effect of PTEN deficiency during the NSC transformation, PTEN $-1-$ NSCs were transduced with lentiviral particles encoding two independent PAX7 shRNAs, which resulted in downregulation of PAX7 in the PTEN $-1-$ NSCs (Supplementary Fig. 12d). Silencing PAX7 effectively inhibited the clonal expansion and migratory capabilities of $P T E N^{-1-}$ NSCs in vitro, as well as the intracranial neoplasm formation in vivo (Fig. 4c,d and Supplementary Fig. 12d-h). Ectopic expression of PAX7 dominant-negative mutants, which do not contain the transactivation domain, also inhibited the neoplastic features of $P_{T E N^{-1}}$ - NSCs (Fig. 4e), suggesting a critical role of the transcriptional activity of PAX7 in promoting neoplastic phenotypes in NSCs. To examine if elevated level of PAX7 alone is able to cause the neoplastic changes in WT NSCs, we overexpressed PAX7 in WT NSCs, to a similar level as in $P_{T E N}{ }^{-1-}$ NSCs, and found that ectopic PAX7 expression recapitulated the major phenotypes caused by PTEN depletion, including an increase of in vitro cellular migration and in vivo neoplastic expansion (Fig. 4f and Supplementary Fig. 13a-c). In addition, overexpression of $P A X 7$ in WT NSCs resulted in a gene expression signature resembling that of $P T E N^{-1-}$ NSCs and distinct from WT NSCs (Supplementary Fig. 13d,e and Supplementary Data 3). We found that majority of genes (19/23) whose transcripts were co-upregulated by $P A X 7$ overexpression and PTEN-knockout are associated with tumour-promoting or increased cellular mobility (Supplementary Fig. $13 \mathrm{f}$ and Supplementary Data 3). Therefore, PAX7 appears to be one of the key factors mediating neoplastic potential of PTEN-deficient NSCs.

Given that PTEN was predominantly localized to the nucleus in NSCs (Fig. 1e), and emerging evidence suggests that PTEN associates with chromatin DNA and is involved gene transcription regulation ${ }^{27,44}$, we thus reason that PTEN may repress $P A X 7$ transcription through direct binding to its promoter. A ChIP-based promoter mapping revealed a 981-bp DNA region upstream of the transcription starting site (TSS) of $P A X 7$ as being a docking site for PTEN in WT NSCs, but not in WT MSCs (Fig. 4g and Supplementary Fig. 14a). When cloned in a luciferase reporter, this region showed higher cis-activation ability when PTEN was depleted in NSCs (Fig. 4h). Bioinformatics analysis predicted several binding sites in this region for transcription factor CREB and its co-activator CBP/p300 (http://www.cbrc.jp/research/db/TFSEARCH.html). Given that CREB is a direct phosphatase substrate of nuclear PTEN ${ }^{45}$, we hypothesized that interplay between PTEN and CREB account for the PTEN-mediated transcriptional repression of $P A X 7$ as well as other genes in NSCs. Supporting this hypothesis, $P T E N^{-/}$NSCs demonstrated elevated levels in the phosphorylated form of CREB (p-CREB; Fig. 4i). Bioinformatics predicted that $167(51.2 \%)$ of 326 genes that were upregulated in $P T E N^{-1-}$ NSCs are potentially CREB targets $\left(P<10 \mathrm{E}^{-4}\right.$; Fig. $\left.4 \mathrm{j}\right)$. Among these genes, the promoters of 108 genes showed clear enrichments of the active histone mark $\mathrm{H} 3 \mathrm{~K} 4 \mathrm{me}$, suggesting a permissive/active gene transcription state (Fig. 4j, Supplementary Fig. $14 \mathrm{~b}$ and Supplementary Data 4). As for PAX7 gene, ChIP-quantitative-PCR showed that in WT NSCs PTEN and CREB/CBP co-occupied within the 981-bp DNA region at the $P A X 7$ promoter (Fig. 4k). Co-immunoprecipitation (IP) and ChIP analyses further indicated that PTEN formed a protein complex with CREB and CBP (Supplementary Fig. 14c,d) ${ }^{45,46}$. Moreover, depletion of PTEN in WT NSCs resulted in an elevated occupation of $\mathrm{p}$-CREB and/or $\mathrm{CBP}$ as well as $\mathrm{H} 3 \mathrm{~K} 4 \mathrm{me} 3$ at the PAX7 promoter (Fig. 4k and Supplementary Fig. 14b). Using a GAL4-based reporter assay, we found that the co-activator activity of CBP was elevated in PTEN-deficient NSCs, which could be rescued by reintroduction of PTEN (Supplementary Fig. 14e). These results support a model that PTEN deficiency led to a de-repression of CREB/CBP machinery at the PAX7 promoter, resulting in activation of $P A X 7$ transcription (Supplementary Fig. 14f).

Next, we investigated whether over-activation of CREB/CBP upon PTEN deficiency could underlie the neoplastic transformation of NSC. For this purpose, we first repressed CBP/p300 activity in $P T E N^{-1-}$ NSCs by shRNA. Silencing CBP/p300 effectively decreased the migration of PTEN-deficient NSCs (Supplementary Fig. 14g,h). In addition, we compared the abilities of the WT, protein phosphatase-dead mutant (Y138L) and lipid phosphatase-dead PTEN mutant (G129E) of PTEN in rescuing the malignant phenotypes of $P T E N^{-1-}$ NSCs. Although complementation with either the WT or the G129E mutant PTEN effectively rescued the neoplastic phenotypes in PTEN $^{-1-}$ NSCs, mutation of PTEN at Y138L resulted in loss of rescue ability (Fig. 4l), which is consistent with its inability to dephosphorylate CREB (Supplementary Fig. 14i). These observations indicated that the neoplasm-suppressing activity of PTEN in NSCs is at least in part dependent on its ability of transrepressing CREB/CBP.

Together, these data support a model that PTEN functions as a trans-repressor at the PAX7 gene promoter in NSCs; PTEN deficiency relieves the repression and activates CREB/ CBP-mediated transcription of $P A X 7$ during the neoplastic transformation of NSCs.

The PTEN-PAX7 axis regulates malignant features of GBM. Our results support the view that human NSCs are the potential cell-of-origin for GBM, especially in the context of PTEN deficiency. It is perceivable that GSCs, the tumorigenic population within GBM, inherit genetic trait from mutated NSCs that potentially mediates the malignancy of GBM. We established three lines of GSCs from primary patient GBM specimens based on the established methods ${ }^{30,47}$. These GSCs exhibited features characteristic of NSCs and could be cultured under neurosphere or monolayer conditions (Fig. 5a,b, Supplementary Fig. 15a,b and Supplementary table 1$)^{30}$. Western blotting and immunofluorescence showed that these GSCs expressed normal levels of PTEN protein (referred to as parental GSCs; Supplementary Fig. 15c). To create isogenic GSCs that are deficient for endogenous PTEN (referred to as GSCs-PTEN ${ }^{\text {low }}$ ), the parental GSCs were transduced with a lentiviral vector encoding PTEN shRNA. Silencing PTEN in the parental GSCs resulted in a marked increase of migration capability (Fig. $5 \mathrm{c}$ and Supplementary Fig. 15d). Correspondingly, GSCs-PTEN ${ }^{\text {low }}$ exhibited increased levels of phosphorylated AKT, phosphorylated CREB and PAX7 (Supplementary Fig. 15e,f). Knockdown of CBP/p300 in GSCs-PTEN ${ }^{\text {low }}$ not only impaired PAX7 expression but also compromised cellular migration potential (Supplementary Fig. 15g,h), whereas overexpression of PAX7 in the parental GSCs resulted in a more aggressive migratory phenotype (the resulting cells referred to as GSCs-PAX7 $\left.7^{\text {high }}\right)$, similar to that observed in GSCs-PTEN ${ }^{\text {low }}$ (Fig. 5d and Supplementary Fig. 15d,i).

To establish a connection between PTEN and PAX7 in human GBMs, we examined the Cancer Genome Atlas (TCGA) data set of $532 \mathrm{GBMs}^{48}$, and found that GBMs with decreased PTEN expression demonstrated increased $P A X 7$ transcript (Spearman correlation coefficient $=-0.28, P$ value $=1.0 \times 10^{-11}$; Fig. 5e), therefore providing a strong evidence that PTEN expression negatively correlates with $P A X 7$ transcripts in clinical GBM settings. 
a

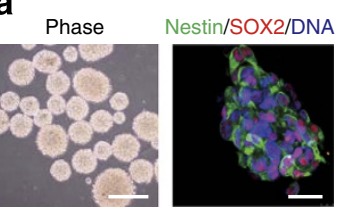

Sphere culture

C

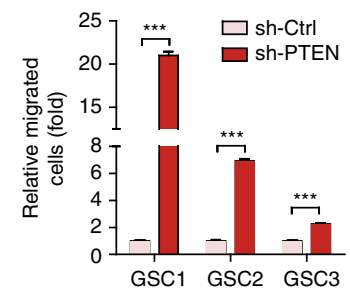

Phase

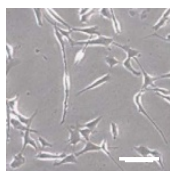

Monolayer culture

d

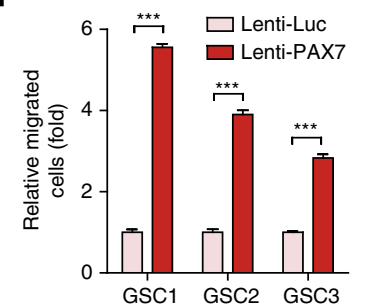

b

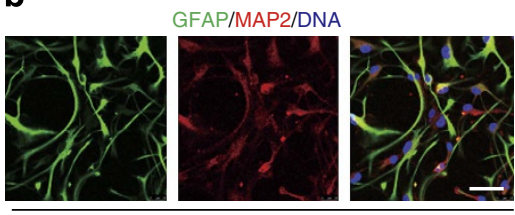

Differentiation

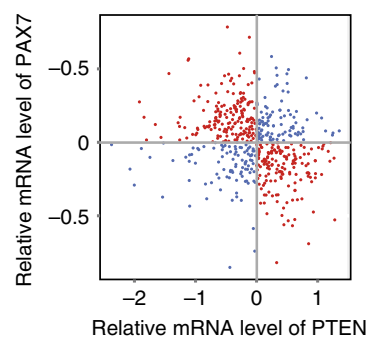

$\mathbf{f}$
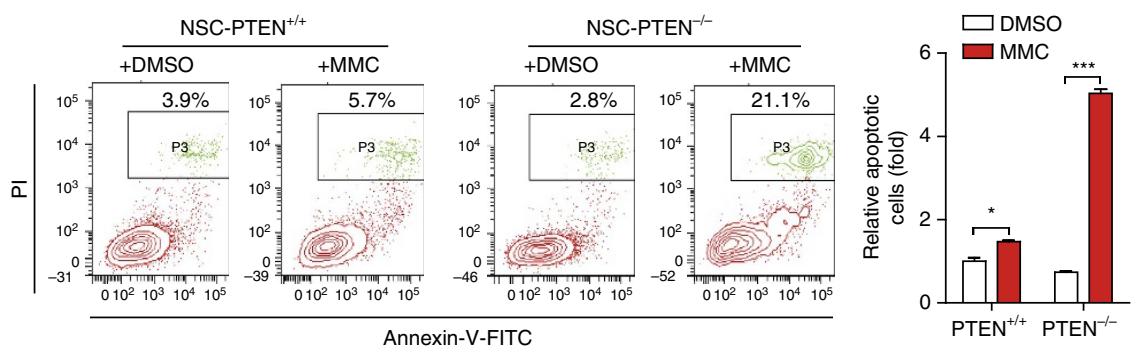

g

h
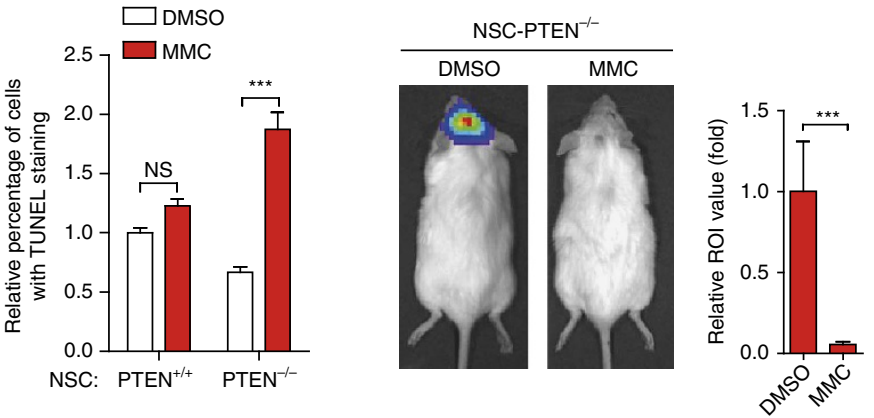

Figure 5 | PTEN-PAX7 axis functions in GSCs and GBMs. (a,b) Immunofluorescence analysis of the indicated antigens in GSCs (a) and their spontaneously differentiated derivatives (b). Scale bars, $1 \mathrm{~mm}$ (phase) and $25 \mu \mathrm{m}$ (IF). (c,d) Cell migration assay indicating increased cellular mobility in PTEN-knocked down (c) or PAX7-overexpressed (d) GSC lines. $n=3$. ${ }^{\star \star \star} P<0.001$ (t-test). (e) GBM microarray data were obtained from TCGA website and spearman correlation was calculated by all the samples which were plotted by red and blue dots. The gene expression level was log2 lowess normalized. PAX7 expression negatively correlated with PTEN (Spearman correlation coefficient $=-0.28, P$ value $=1.0 \times 10^{-11}$ ). (f,g) FACS $(\mathbf{f})$ and TdT-mediated dUTP nick end labelling (g) analyses of cell apoptosis in MMC $(7.5 \mu \mathrm{M}, 24 \mathrm{~h})$ or vehicle-treated WTand PTEN ${ }^{-/}-\mathrm{NSC}$. $n=6$. ${ }^{*} P<0.05$ ( $t$ test); ${ }^{\star \star \star} P<0.001$ ( $t$-test); NS, not significant. (h) Representative bioluminescent images of PTEN ${ }^{-/}-$NSCs implanted animals in the presence or absence of MMC treatment are shown at day 70 after injection (left). Right panel indicates average bioluminescent signals. $n=5$ for dimethylsulphoxide (DMSO) treatment, and $n=8$ for $M M C$ treatment. ${ }^{\star \star \star} P<0.001$ (t-test).

Mitomycin C (MMC) triggers apoptosis in PTEN-deficient NSCs. Given that the PTEN deficiency is associated with increased metastasis, recurrence and drug resistance in GBM patients, discovery of compounds selectively killing PTEN-deficient GSCs may represent an important step forward towards precision therapy on GBM patients with known PTEN mutation(s). We therefore sought to utilize the isogenic NSC platform to screen compounds specifically kill PTEN-deficient NSCs while not the WT NSCs. By testing compounds with reported tumour-killing activity (Supplementary Table 2), we identified MMC, a clinically used anti-tumour agent with DNA-crosslinking activity ${ }^{49}$, as a potential candidate compound. MMC selectively killed
PTEN $^{-1-}$ NSCs while showed minimal effect on WT NSCs, as evidenced by propidium iodide staining and TdT-mediated dUTP nick end labelling assays (Fig. 4f,g and Supplementary Fig. 16a). Temozolomide, a clinically used anti-GBM drug, however, had a comparable effect on WT and PTEN ${ }^{-1-}$ NSCs (Supplementary Fig. 16b). MMC was also found cytotoxic to NSCs overexpressing PAX7 (Supplementary Fig. 16c).

To evaluate the efficacy of MMC in vivo, we performed intracranial administration of $\mathrm{MMC}$ to mice harbouring neoplasm derived from $P T E N^{-1-}$ NSC engrafts. MMCtreatment effectively repressed neoplastic growth from the PTEN $^{-/-}$NSC engrafts (Fig. 5h). 


\section{Discussion}

GBM is one of the most deadly forms of human cancers and has a median survival time of only $\sim 14.6$ months. Up to date, most studies of GBM pathogenesis rely on GBM cell lines, primary GSCs or GBM cells from fresh specimens, or mouse GBM models. These conventional source materials have undoubtedly aided in our understanding of GBM pathogenesis and facilitated development of novel therapies, however, have their drawbacks. For instance, GBM or GSC lines bear significant variations from line to line, such as marker expressions, differences in cellular behaviours in vitro or in vivo, and sensitivities to drugs. These differences can be attributed to different culture conditions, pathological stages or, more likely, from their distinct genetic backgrounds. The complex genetic mutations, including the primary, secondary or evolved ones in tumour suppressors and oncogenes, as well as their interactions constitute a roadblock for studying the oncogenic identity and cell-of-origin for GSCs, as well as for aetiological understanding of GBM. Although it has been well established that GSCs are directly linked to NSCs, it has been challenging to study how genetic mutations may contribute to malignant transformation of NSCs as well as initiation and progression of GBM. The primary GBM subtype is acute and of high-grade and frequently harbours loss-of-function mutations in PTEN gene. Mouse models have been employed to study the role of PTEN deficiency in NSCs ${ }^{13,50,51}$. Given that species-difference exists between mouse and human, a human cellular platform to study GBM origin or pathogenesis associated with PTEN deficiency or other oncogenic events is thus urgently needed. It was not until very recently that two elegant human intestine tumour models generated using CRISPR/Cas9-based gene editing were reported ${ }^{25,26}$. Nevertheless, none of above-mentioned models investigated tumorigenesis in a genuine tumourinitiating niche. Our study for the first time uses genetically engineered human NSCs to study a specific genetic mutation on neoplastic transformation both in vitro and in intracranial tumour-initiating niches, and provided the proof-of-concept evidence that NSC is a most susceptible human adult stem cell type for PTEN-deficiency-induced oncogenic transformation. Nevertheless, it should be emphasized that GBM are frequently associated with more than one genetic mutations. Indeed, compared with the GSCs derived from clinically obtained GBM samples that potentially harbour multiple genetic mutations ${ }^{52}$, PTEN-deficient human NSCs show milder phenotypes in intracranial implantation assay, and do not show aggressive growth in non-neural microenvironment. We have shown that p53 insufficiency and PTEN deficiency resulted in a cooperative effect on NSC transformation, which is consistent with a recent mouse study indicating that simultaneous inactivation of Pten and p53 in mouse neural stem/progenitor cells led to malignant transformation and tumour formation ${ }^{13}$. From this point of view, the isogenic NSC platform also provides a useful model to study other individual neoplastic mutations, or their combinations in mediating malignant human NSC transformation.

Accumulating evidence indicated that PTEN plays a role in the nucleus through protein-protein interactions ${ }^{27,44}$. We showed here that PTEN is a nucleus-localized protein in human NSCs. It associates with and inactivates $\mathrm{CREB} / \mathrm{CBP}$ at $P A X 7$ promoter, and mediates transcriptional repression of PAX7. To our knowledge, this is the first report to show that PTEN can trans-repress gene expression via the association with transcriptional activators on gene promoters. Notably, a recent report suggests that PTEN binds to Rad51 promoter and is required for its expression in $\mathrm{MEFs}^{27}$. In a separate study, PTEN is identified to be associated with histone $\mathrm{H} 1$ and accounts for maintenance of a condensed chromatin configuration ${ }^{44}$. Our findings, together with these observations, support the notion that nuclear PTEN may act as a key transcriptional regulator in a context-dependent manner. Given that $P A X 7$ is among the earliest genes involved in neural progenitor commitment in the embryo, the elevated expression of PAX7 in PTEN-deficient GSCs may account for the low-differentiated histology of GBM, a common feature for more aggressive cancers. Together with the finding that PTEN is negatively correlated with PAX7 in clinical human GBM samples, it is likely that the overexpression of PAX7 in GSCs is one of the key factors mediating GBM tumorigenesis or malignancy.

Owing to its prevalence in GBM and other cancers, PTEN mutations have been regarded as a major target for drug discovery. Cell-based systems are advantageous for automate chemical screening or drug evaluation. Most of previous studies rely on cancer cells or primary cultures and use non-isogenic cells as control, which are limited by confounding effect of different genetic backgrounds. When it comes to screening for specific drug to kill GSCs, human fetal NSCs from a different genetic background are the common cells to be used as a control ${ }^{53}$. Our isogenic PTEN-knockout NSCs recapitulated certain features of GSCs, thus holding the potential to be used as a platform for screening 'precision drugs' against GSCs based on 'PTEN deficiency'. We found that MMC is a candidate compound, and preferentially targeting PTEN-deficient NSCs while sparing their PTEN-proficient counterparts. We further showed that transcranial injection of MMC could diminish neoplasia from PTEN-deficient NSCs. Nevertheless, although MMC is used in the clinic for other cancers, it does not cross the blood-brain barrier and has not been used for GBM. Thus, developing advanced MMC derivatives being capable of passing through blood-brain barrier and with reduced toxicity may represent a potential therapeutic strategy for GBM harbouring PTEN mutations, despite that the underlying pharmacological mechanism warrants further investigations. To our best knowledge, this is the first study using isogenic NSCs bearing a genetically engineered carcinogenic mutation as a potential drug discovery strategy. We propose here a simplified approach to examine 'one mutation' at a time, using human ESC (or iPSC)-derived NSCs with a well-known genetic background to screen or evaluate drugs being capable of targeting certain genetic mutations.

\section{Methods}

Antibodies and reagents. The following commercial primary antibodies were used: anti-PTEN (9559; Western blotting (WB),1:1,000; immunofluorescence (IF), 1:200), anti-Phospho-PTEN (pSer380, 9551, 1:1,000), anti-Phospho-AKT (pSer473 4060, WB,1:1,000;IF, 1:200), anti-Phospho-AKT (pThr308, 2965, 1:1,000), antiPhospho-GSK-3 $\beta$ (pSer9, 5558, 1:1,000), anti-Phospho-mTOR (pSer2448, 5536, 1:1,000), anti-phospho-CREB (pSer133, 9198, 1:1,000), anti-CREB (9197, 1:1,000) antibodies were from Cell Signaling Technology; anti-NANOG (ab21624, 1:250), anti-Ki67 (ab16667, 1:1,000), anti-Nucleolin (ab22758, 1:200), anti-CBP (ab2832, 1:1,000) antibodies were from Abcam; anti-OCT4 (sc-5279, 1:100), anti-SOX2 (sc17320, 1:100), anti-Actin (sc-130301, 1:2,000), anti-GFAP (sc-9065, 1:1,000), antiTRA-1-81 (sc-21706, 1:20) antibodies were from Santa Cruz Biotechnology; antiNestin (MAB5326,1:500), anti-Human nuclei (MAB1281, 1:1,000) antibodies was from Millipore; anti-MAP2 (m4403, 1:500), anti-Tuj1 (T2200, 1:500), anti-Flag (M2, 1:2,000), anti-SMA (A5228, 1:100), anti-AFP (A8452, 1:200), anti-tubulin (T5168, 1:2,000) antibodies were from Sigma; anti-CD34 (555822, 1:50), anti-CD43 (560198, 1:50), anti-CD45 (555482, 1:50), anti-CD73 (550741, 1:50), anti-CD90 (555595, 1:100) antibodies were from BD Biosciences; anti-CD105 (17-1057, 1:100) antibody was from eBioscience; anti-PAX6 (PRB-278P, 1:500) antibody was from Covance; anti-PAX7 antibody (WB, 1:500; IF, 1:50) was from Atsushi Kawakami; anti-CD133 (130-080-801, 1:10) was from Miltenyi Biotec; MMC was purchased from Biomol International. Temozolomide was purchased from Sigma.

Cell culture. H9 ESCs were purchased from WiCell Research Institute and cultured as previously described ${ }^{54}$. Briefly, human fibroblast-derived iPSCs, H9 ESCs and their PTEN knockout derivatives were maintained on MMC inactivated MEFs in human ESC culture medium; whereas the feeder-free cultures were maintained on Matrigel- (BD Biosciences) coated plates in mTeSR medium (STEMCELL 
Technologies). The human ESC culture medium contains: 80\% DMEM/F-12 (DMEM; Invitrogen), 20\% knockout serum replacement (Invitrogen), 1\% GlutaMAX (Invitrogen), 1\% non-essential amino acids (Invitrogen), 1\% PenicillinStreptomycin (PS, Invitrogen), $55 \mu \mathrm{M} \beta$-mercaptoethanol and $10 \mathrm{ng} \mathrm{ml}^{-1}$ of human basic fibroblast growth factor (bFGF; Joint Protein Central). MSCs were grown on Gelatin-coated plates in culture medium containing 90\% $\alpha$-MEM (Invitrogen), $10 \%$ fetal bovine serum (AusGeneX), $1 \%$ non-essential amino acid, $1 \%$ PS and $1 \mathrm{ng} \mathrm{ml}^{-1}$ of bFGF. NSCs were cultured in Neural Stem cell Maintenance Medium containing $50 \%$ Advanced DMEM/F12, 50\% Neurobasal, $1 \times$ N2, $1 \times$ B27, 2 mM GlutaMAX, $10 \mathrm{ng} \mathrm{ml}^{-1}$ of human leukemia inhibitory factor (hLIF), $3 \mu \mathrm{M}$ CHIR99021 and $2 \mu \mathrm{M}$ SB431542. GSCs were grown in medium containing 90\% Neurocult NSC Basal Medium (STEMCELL Technologies), 10\% NeuroClut NSC Proliferation Supplements (STEMCELL Technologies), $20 \mathrm{ng} \mathrm{ml}^{-1}$ of EGF and $20 \mathrm{ng} \mathrm{ml}^{-1}$ of bFGF and $2 \mu \mathrm{g} \mathrm{ml}^{-1}$ of heparin ${ }^{55}$. HEK293T cells were maintained in high-glucose DMEM containing $10 \%$ fetal bovine serum (FBS, Gemini). No mycoplasma contamination was observed during cell culture.

Generation of PTEN knockout (PTEN ${ }^{-/}{ }^{-}$) human ESCs. TALENs against the human PTEN gene (PTEN-TALEN_Left and PTEN-TALEN_Right) were purchased from Addgene (Plasmid \#36764 Plasmid \#36765). The donor plasmid for HR was described previously 22 . The following PCR primers were used for cloning the homology arms into the donor vector (PTEN-HR-Neo-pCR2.1):

Left-F: $5^{\prime}$-CGGGGTACCGACCGTCCCTGCATTTCCCTCTACACT-3';

Left-R: $5^{\prime}$-CGCGGATCCCGGAATGGGGAGAAGACGAATAATCCTCC

GAAC- $3^{\prime}$;

Right-F: 5'-ACGCGTCGACCCTGGTTGCAAGTGTCAA GCCACCGAT-3';

Right-R: 5'-ATAAGAATGCGGCCGCCCCCATCCCTAATCAAAACCAATG

TGTTGTACCTG-3';

H9 ESCs were pre-treated with ROCK inhibitor Y-27632 (Sigma) $24 \mathrm{~h}$ before plasmid electroporation. On the day of electroporation, H9 ESCs were individualized with TrypLE (Invitrogen) and filtered through a 40- $\mu \mathrm{m}$ cell strainer to remove cell clumps. For TALEN-mediated engineering of $P T E N^{-1-}$ ESC lines, $10^{6}$ cells were counted and resuspended in $1 \mathrm{ml}$ of MEF-conditioned medium containing $10 \mu \mathrm{M}$ ROCK inhibitor Y-27632, then mixed with plasmids $(8 \mu \mathrm{g}$ PTEN-TALEN_Left, $8 \mu \mathrm{g}$ PTEN-TALEN_Right, $2 \mu \mathrm{g}$ Neo-PTEN-HR-pCR2.1 donor vector) and electroporated. After electroporation, cells were plated on MMC inactivated G418-resistent MEF cells. Two days later, cells were treated with $50 \mu \mathrm{g} \mathrm{ml}^{-1}$ of G418 (Gibco) for selection. After 14-21 days of selection, G418-resistant clones were manually picked and transferred to 96-well plates and expanded for genotyping. Gene-targeting was determined by PCR-screening of genomic DNA from the drug-resistant clones with the following primers:

P1: 5'-AGGCTGTTACAGTCAAATCTCTGCGAACGAT-3';

P2: $5^{\prime}$-CCCCAAAGGCCTACCCGCTTCCATTGCTCA- ${ }^{\prime}$;

P3: 5'-CTACCTGCCCATTCGACCACCAAGCGAAACATC-3';

P4: 5'-TGTCCACTGCCACAATTCGCATTACCAAACTCA-3'

Homozygous deletion of exon 1 of the PTEN gene was verified by a two-step

PCR with the following primers:

P5: 5'-CCCAGACATGACAGCCATCATCAAAGAGATCG-3';

P6: 5'-AAAACGGTGAGCAGCAATTCTGGCATCACA-3';

P7: 5'-TTTGGGCCCCACACAGTGCAAGCTAAAGCAACCCCGTCT-3';

P8: 5'-CCGCTCGAGAACATCACTGGTCCTGGGCAAAATCGAGA- ${ }^{\prime}$.

The PTEN locus was further examined by Southern blotting after extracting genomic DNA from PCR-positive clones. The neomycin-resistance cassette was removed according to the previously described method ${ }^{22,23}$. Briefly, in order to remove the neomycin-resistance cassette, PTEN knock-out hESCs $\left(5 \times 10^{6}\right.$ cells $)$ were electroporated with pCAG-Flpo-2A-puro vector $(10 \mu \mathrm{g})$, and then plated on MMC-inactivated DR4-MEF cells. Two days later, puromycin $\left(1 \mu \mathrm{g} \mathrm{ml}^{-1}\right.$.

Invitrogen) was added to the medium to enrich Flpo recombinase-expressed cells. The emerging colonies were expanded, and the removal of the neomycin-resistance cassette was verified by PCR using the following primers: P1: $5^{\prime}$-AGGCTGTTA CAGTCAAATCTCTGCGAACGAT- $3^{\prime}$ and P4: $5^{\prime}$-TGTCCACTGCCACAAT TCGCATTACCAAACTCA-3'.

Flow cytometry analysis. For TRA-1-81 analysis, each $100 \mu$ of cell suspension $\left(10^{6}\right.$ cells) was used. Cells were incubated with the primary antibody mouse anti-TRA-1-81 (Santa Cruz) and then the secondary Alexa Fluor 568-labelled Goat anti-Mouse IgM (Invitrogen). For CD133 analysis, each $100 \mu \mathrm{l}$ of cell suspension $\left(10^{6}\right.$ cells) was used. Cells were incubated with the primary antibody CD133/1(AC133)-PE (Miltenyi Biotec). For cell cycle analysis, cells were processed with the Click-iT EdU Flow Cytometry Assay Kits (Invitrogen) according to the manufacturer's instructions. Briefly, the cells were harvested after incubation with EdU $(10 \mu \mathrm{M})$ in medium. $100 \mu \mathrm{l}$ of cell suspension $\left(10^{6}\right.$ cells) for each sample were used. For the detection of cell apoptosis, each sample ( $10^{6}$ cells) was subjected to Annexin V-PE staining ${ }^{20}$. Cells were examined by fluorescence-activated cell sorting (FACS) using a flow cytometer (FACSort; Becton, Dickinson and Company), and the cell cycle populations were determined using ModFit software (Verity Software House, Inc.).

Generation and characterization of NSCs. Neural induction was based on previous reports ${ }^{20,22}$. In brief, ESCs cultured on MEF feeder cells were challenged with
NIM-1 medium (50\% Advanced DMEM/F12 (Invitrogen), 50\% Neurobasal (Invitrogen), $1 \times \mathrm{N} 2$ (Invitrogen), $1 \times$ B27 (Invitrogen), $2 \mathrm{mM}$ GlutaMAX (Invitrogen) and $10 \mathrm{ng} \mathrm{ml}^{-1}$ of hLIF (Millipore), $4 \mu \mathrm{M}$ CHIR99021 (Cellagentech), $3 \mu \mathrm{M}$ SB431542 (Cellagentech), $2 \mu \mathrm{M}$ Dorsomorphin (Sigma) and $0.1 \mu \mathrm{M}$ Compound E (EMD Chemicals Inc.). Two days later, medium was switched to NIM-2 (50\% Advanced DMEM/F12, 50\% Neurobasal, $1 \times$ N2, $1 \times$ B27, $2 \mathrm{mM}$ GlutaMAX, $10 \mathrm{ng} \mathrm{ml}^{-1}$ of hLIF, $4 \mu \mathrm{M}$ CHIR99021, $3 \mu \mathrm{M} \mathrm{SB} 431542$ and $0.1 \mu \mathrm{M}$ Compound E) for additional 5 days. The cultures were then split onto Matrigelcoated plates and maintained in neural stem cell maintenance medium (NSMM) containing $50 \%$ Advanced DMEM/F12, 50\% Neurobasal, $10 \mathrm{ng} \mathrm{ml}^{-1}$ of hLIF, $1 \times$ N2, $1 \times$ B27, 2 mM GlutaMAX, $3 \mu \mathrm{M}$ CHIR99021 and $2 \mu \mathrm{M}$ SB431542.

Neuronal differentiation assay. $1 \times 10^{5}$ NSCs cultured on Matrigel-coated one well of six-well plate were switched to neuronal differentiation medium containing DMEM/F12, $1 \times$ N2, $1 \times$ B27, $200 \mu$ M Ascorbic acid (Sigma), $400 \mu$ M dbcAMP (Sigma), $10 \mathrm{ng} \mathrm{ml}^{-1}$ of GDNF (Peprotech) and $10 \mathrm{ng} \mathrm{ml}^{-1}$ of BDNF (Peprotech). Laminin (Sigma) was used to facilitate differentiation. Cells were cultured for 21 days, and then characterized by MAP2 and Tuj1 immunostaining ${ }^{20,22}$.

Bisulfite sequencing of the OCT4 promoter. Bisulfite conversion of DNA was carried out using CpGenome Fast DNA Modification Kit (Millipore) following the manufacturer's recommendation. About $1 \mu \mathrm{g}$ of DNA was used as starting material. A genomic fragment of the OCT4 promoter was amplified using LA Taq Hot Start Version (TAKARA) as previously described ${ }^{56}$. In brief, PCR products were purified by gel extraction using QIAquick columns (Qiagen), and subsequently cloned into the pEasy-T1 vector (Transgen). Thirteen clones from each sample were sequenced with the universal primer M13.

Derivation of MSCs from ESCs. MSCs were differentiated from ESC lines as previously described ${ }^{22,23}$. In brief, groups of 10-14 EBs were plated on Matrigelcoated six-well plates in $\alpha$-MEM (Invitrogen) medium with 10\% FBS (Hyclone), $1 \%$ PS, $10 \mathrm{ng} \mathrm{ml}^{-1}$ of bFGF and $5 \mathrm{ng} \mathrm{ml}^{-1}$ of TGF $\beta$ (HumanZyme). Cells were left for about 10 days until confluent MSC-like populations occurred. The resulting cells were passaged once and then analysed and sorted by FACS using various antibodies related to the MSC signatures. The cells after sorting were set to passage 0 (P0). Expressions of MSC-specific markers were verified by immunostaining with antibodies against CD73 (BD), CD90 (BD) and CD105 (eBioscience).

Isolation and culture of human GSCs. Three glioblastoma samples were obtained in the Beijing Tiantan Hospital, following approval from the institutional review board, and written informed consent was obtained from all patients. GSCs were isolated according to previously described methods with minor modifications ${ }^{30,55}$ Briefly, tumour tissues were dissociated into single cells with Accumax Cell Dissociation Solution (Millipore) for $30 \mathrm{~min}$ at $37^{\circ} \mathrm{C} .2 \times 10^{6}$ single cells were initially seeded into one well of a six-well plate to allow formation of spheres/aggregates. In addition to sphere culture, GSCs were also cultured as monolayer in Matrigel-coated culture dishes. Cells were stably maintained either as spheres or as monolayer culture.

Neurosphere assay. $2 \times 10^{4}$ single NSCs were seeded into one-well of an ultralow attachment 96-well plate (Corning) to allow neurosphere formation. Medium was changed every two days. The diameters of neurospheres were measured using Image J.

Luciferase reporter assays. A 981-bp genomic DNA fragment containing the PTEN docking site upstream of the TSS of $P A X 7$ was cloned into the pGL3Promoter vector (Promega). The DNA fragment was amplified by PCR using the following primers: 5' -CGGGGTACCCAAGTCTGGCCCAGCCCACACACTCA G-3' (forward primer); 5'-CCGCTCGAGGCCCTCCCCCGGGAGAGATTGG GAGATC- $3^{\prime}$ (reverse primer). Transient transfection with the luciferase reporter plasmids was performed in 12-well cell culture plates according to the manufacturer's protocol. Cells were lysed $48 \mathrm{~h}$ post transfection, and firefly and Renilla luciferase activity were measured with a Synergy H1 Hybrid Reader(BioTek), and Renilla was used as an internal control.

Immunofluorescence microscopy. Cells were fixed with $4 \%$ formaldehyde in PBS at room temperature (RT) for $20-30 \mathrm{~min}$. After fixation, cells were permeabilized with $0.4 \%$ Triton X-100 in PBS for 5 min at RT. After incubation with $10 \%$ FBS or BSA in PBS for $30 \mathrm{~min}$, cells were incubated at RT for $1 \mathrm{~h}$ or at $4{ }^{\circ} \mathrm{C}$ overnight with the primary antibody. Subsequently, incubation at RT for $20 \mathrm{~min}$ with the corresponding secondary antibody was carried out. Nuclei were stained with Hoechst 33342 (Invitrogen). A Leica SP5 confocal was used for Immunofluorescence microscopy.

Teratoma analysis. Teratoma assay was performed as described ${ }^{57}$. In brief, $3 \times 10^{6}$ ESCs were administrated subcutaneously into NOD/SCID mice (male, 6-8 weeks). Mice were killed 6-12 weeks after injection, and teratomas were analysed 
by immunofluorescence staining. All animal experiments were conducted with approval of the Institute of Biophysics, Chinese Academy of Science.

SA-p-Gal staining. Staining was conducted as described previously ${ }^{23}$. In brief, cells were fixed at RT in $2 \%$ formaldehyde and $0.2 \%$ glutaraldehyde for $3 \mathrm{~min}$. Fixed cells were then stained with fresh staining solution at $37^{\circ} \mathrm{C}$ overnight. The percentage of cells positive for SA- $\beta-$ Gal signal were quantified and statistically analysed.

Lentivirus production. Expression and purification of lentiviruses were performed as previously described with minor modification ${ }^{23}$. For generation of lentiviral vectors encoding shRNA targeting PTEN, PAX7 or CBP/p300, corresponding shRNA oligos (Supplementary Table 3) were cloned into the PLVTHM vector pre-cleaved by ClaI and MluI. For lentiviral vectors expressing PTEN, PAX7 or luciferase (control), corresponding cDNAs were cloned into the pLE4 vector (a kind gift from Dr Tomoaki Hishida). For lentiviral packaging, HEK293T cells were co-transfected with lentiviral expression or shRNA vectors, as well as psPAX2 (Addgene, 12260) and pMD2.G (Addgene, 12259). Lentiviral particles were collected after $48 \mathrm{~h}$ and concentrated by ultracentrifugation at $19,400 \mathrm{~g}$ for $2.5 \mathrm{~h}$. Concentrated viruses were used for titre determination and cell transduction.

Western blotting, IP and RT-qPCR. For Western blotting, cells were lysed in RIPA buffer ( $25 \mathrm{mM}$ Tris-HCl pH 7.6, $150 \mathrm{mM} \mathrm{NaCl}, 1 \% \mathrm{NP}-40,1 \%$ sodium deoxycholate, $0.1 \%$ SDS) with protease inhibitor cocktail (Roche). A BCA kit (Thermo Fisher Scientific) was used for protein quantification. About $20 \mu \mathrm{g}$ of protein lysate was subjected to SDS-PAGE electrophoresis and electrotransferred to a PVDF membrane (Millipore). Then primary and HRP-conjugated secondary antibodies were used to visualize the target proteins. The quantification of western blot was performed with Image Lab software for ChemiDoc XRS system (Bio-Rad). For IP, $\sim 1 \times 10^{7}$ cells was lysed on ice for $1 \mathrm{~h}$ in NP40 lysis buffer $(50 \mathrm{mM}$ Tris-HCl, pH 8.0, 1\% NP-40, $150 \mathrm{mM} \mathrm{NaCl}, 2 \mathrm{mM}$ EDTA, 1 mM PMSF, Complete EDTA-free Protease Inhibitor Cocktail). After adjusting protein concentrations to about $4 \mathrm{mg} \mathrm{ml}^{-1}$, inputs were aliquoted, and lysates were incubated with anti-Flag agarose (Sigma) overnight at $4{ }^{\circ} \mathrm{C}$. After three washes in NP-40 lysis buffer, bound proteins were eluted with $1 \times$ SDS loading buffer, and samples were analysed by western blotting with the indicated antibodies. For RT-qPCR, cellular total RNA was extracted by TRIzol (Invitrogen), and genomic DNA was removed by DNA-free Kit (Ambion), followed by cDNA synthesis with GoScript Reverse Transcription System (Promega). RT-qPCR was performed using iTaq Universal SYBR Green Supermix (Bio-Rad). Primer sequences are given in Supplementary Table 3. The uncropped images of the western blots and gels are shown in Supplementary Fig. 17.

Cell viability assay. Cell viability was determined with CellTiter 96 AQueous One Solution Cell Proliferation Assay MTS (3-(4,5-dimethylthiazol-2-yl)-2,5diphenyltetrazolium).

In vitro cell migration assay. The cell migration of NSCs, MSCs and GSCs through the Matrigel (BD Bioscience) in vitro was performed as previously described ${ }^{58}$. First, the top chamber of the Transwells (24-well insert; pore size, $8 \mathrm{~mm}$; Corning Costar) pre-coated with Matrigel was seeded with $2.5 \times 10^{4}$ cells in $100 \mu$ l basal medium (50\% Advanced DMEM/F12, 50\% Neurobasal for hNSCs, $\alpha$ MEM for hMSCs and GSC culture medium without EGF, FGF2 and Heparin for GSCs). The corresponding complete culture medium for each cell type was put into the bottom chambers. Cells that migrated through the Transwells were stained and counted.

Single-cell clonal expansion assay. To determine the clonal expansion efficiency of NSCs and MSCs, 2,000 cells were seeded in a Matrigel-coated 12-well plate, and cultured until clear cell colonies formed. The relative colony number was then determined by crystal violet staining.

Metabolomics evaluation. Metabolite profiling was carried out at Metabolon with LC-MS and GC-MS methods as described previously ${ }^{59}$. Briefly, $10^{6}$ ESCs $\left(\right.$ PTEN $^{+/+}$versus PTEN $\left.^{-l-}\right)$ and NSCs $\left(\right.$ PTEN $^{+/+}$versus PTEN $\left.{ }^{-l-}\right)$ were pelleted, and five replicates were stored at $-80^{\circ} \mathrm{C}$. Samples were prepared and analysed by using Metabolon's standard solvent extraction methods. $100 \mu \mathrm{l}$ of each sample was extracted by methanol. After centrifugation, the supernatant consisting of metabolites was used for MS and LC-MS analyses. The extracts were equally split into two parts for analysis on LC/MS/MS (Thermo Fisher linear Ion Trap mass spectrometer with a Fourier Transform) and GC/MS (Mat-95 XP mass spectrometer) platforms. Data were analysed with Welch's two-sample $t$-test.

Lactate measurements. Lactate secreted in the culture medium was collected and measured by a colorometric L-Lactate assay kit (Abcam) following the manufacturer's instructions.
Mouse brain in vivo imaging. Before intracranial implanting, NSCs were transduced with lentivirus expressing luciferase. $2 \mu \mathrm{l}$ of a $1 \times 10^{7}$ cells per ml cell suspension in high-glucose PBS were injected to the hemi-striatum using stereotactic injection. The following coordinate parameters were used: antero-posterior $=0$; medio-lateral $=+2.5 \mathrm{~mm}$; dorso-ventral $=-3.5 \mathrm{~mm}$. About 10 weeks post injection, mice were injected into peritoneal cavity with luciferin to trace tumour cells in vivo. The animals were next anaesthetized, and bioluminescence was imaged using the IVIS Lumina system (Perkin-Elmer). Images were taken every $2 \mathrm{~min}$ for a course of $30 \mathrm{~min}$ ( $10 \mathrm{~s}$ exposure per image). The mice brains were also imaged 1 and $2 \mathrm{~h}$ post injection ( $10 \mathrm{~s}$ exposure per image). Images were processed using Living Image software. Regions of interest were drawn based on each cell mass, and the total number of photons was recorded for each region of interest. Immunofluorescence staining and H\&E staining were carried out on $5 \mu \mathrm{m}$ cryostat sections.

Magnetic resonance imaging. MRI for mouse brain was performed on a Bruker's PharmaScan 7 T MRI imaging system. The system is equipped with standard actively shielded gradient system. A 72-mm birdcage resonator was used for excitation, and detection was done with a 30-mm surface coil. T2-weighted images were consecutively acquired by using a rapid-acquisition relaxation enhanced sequence. Animals were anaesthetized before MRI.

RNA isolation and sequencing library construction. A total of $5-10 \times 10^{5}$ cells were used to isolate RNA with RNeasy Mini Kit (Qiagen). 1.5-2 $\mu \mathrm{g}$ of RNA was qualified by Fragment Analyzer (Advanced Analytical) and was used to construct sequencing libraries using the TruSeq RNA Sample Preparation Kit (Illumina) following the manufacture's protocol.

ChIP and sequencing. ChIP-seq was done following a previous protocol with minor modifications $s^{60}$. Briefly, cells were cross-linked by $1 \%$ (vol/vol) formaldehyde for $8 \mathrm{~min}$ at RT and then quenched by $125 \mathrm{mM}$ glycine for $5 \mathrm{~min}$ at RT. Then, the cells were lysed in lysis buffer $(50 \mathrm{mM}$ Tris-HCl, $10 \mathrm{mM}$ EDTA, $1 \% \mathrm{SDS}, \mathrm{pH} 8.0$ ), which was followed by chromatin shearing using a Covaris S2 instrument. The samples were then incubated overnight with Protein A-bead bound with the following antibodies separately: anti-PTEN (CST, 9559), anti-CBP (Abcam, ab2832), anti-phospho-CREB (CST, 9198) anti-H3K4me3 (CST, 9751 s) and anti-H3K27me3 (CST, 9733). Normal rabbit IgG (Santa Cruz, SC-2027) or input was used as a negative control. To obtain the DNA of interest, chromatin with the target modification was eluted in elution buffer $(20 \mathrm{mM}$ Tris- $\mathrm{HCl}, 5 \mathrm{mM}$ EDTA, $50 \mathrm{mM} \mathrm{NaCl}, \mathrm{pH} 7.5$ ), and then incubated with Proteinase $\mathrm{K}$ on a thermomixer at 1,300 r.p.m. for $2 \mathrm{~h}$ at $68^{\circ} \mathrm{C}$. Next, the DNA was extracted and purified via phenol-chloroform-isoamyl alcohol extraction and ethanol precipitation. Samples for sequencing including H3K4me3, H3K27me3 and IgG were constructed into libraries with NEBNext DNA Library Prep Reagent Set for Illumina (NEB) as described by the manufacturer.

ChIP-PCR mapping of PTEN-associated genomic region. We mapped the PTEN-binding site at PAX7 promoter spanning from $-5,000$ to $+3,251$ with ChIP-PCR using different primer sets. In brief, to obtain the DNA region of interest, lysed samples were incubated overnight with Protein A-bead bound to anti-PTEN antibody. Then, extracted DNAs were subjected to PCR amplification of the different genomic regions by using different primer sets (Supplementary Table 3).

Whole genome sequencing. Genomic DNA was extracted from $1 \times 10^{6}$ cells by DNeasy Blood \& Tissue Kit (Qiagen) and sheared into 150-200 bp fragments by Covaris S2 and the quality was analysed via Fragment Analyzer (Advanced Analytical). The sequencing libraries were constructed using NEBNext DNA Library Prep Reagent Set for Illumina (NEB).

RRBS and TA-RRBS. RRBS and TA-RRBS were performed as we previously reported $^{6}$. For RRBS, after digested with MspI, genomic DNA was size-selected and spiked in with $0.5 \%$ fully methylated lambda DNA. DNA was then treated with bisulfite after ligation with pre-methylated adapters and amplified with PfuTurbo Cx Hotstart DNA polymerase (Agilent Technologies). For TA-RRBS, after MspI digestion, 50-600 bp DNA fragments were first gel-selected, and then glucosylated with $\beta$-glucosyltransferase and oxidized by recombinant mouse Tet1 (mTet1) subsequently. The final TA-RRBS libraries were generated after bisulfite treatment and amplification with PfuTurbo Cx Hotstart DNA polymerase as stated above.

RNA-Seq data analysis. We aligned reads from RNA-Seq using TopHat ${ }^{62}$ and Cufflinks $^{63}$ and differentially expressed genes were analysed as previously described $^{64}$. A total of $14,797,14,782,14,030$ and 14,322 genes with FPKM $>0.1$ were detected in PTEN $^{+1+}$ NSC, PTEN $^{-1-}$ NSC, PTEN ${ }^{+9+}$ MSC and PTEN $^{-1-}$ MSC, respectively. 281, 705 genes ( $q$-value $<0.05, P$-value $<0.05, \mathrm{FC}>2$ ) were significantly downregulated and 326, 1,139 genes $(q$-value $<0.05, P$-value $<0.05$, FC $>2$ ) were significantly upregulated in $P T E N^{-l-}$ NSCs and MSCs, respectively. $\mathrm{GO}$ for enrichment of genes was assessed by DAVID ${ }^{65}$. 
ChIP-seq data analysis. Sequencing reads from the ChIP-Seq experiment were mapped to the human reference genome (hg19) by Burrows-Wheeler Aligner $(\mathrm{BWA})^{66}$, retaining only unique non-duplicate reads without mismatches in the first $15 \mathrm{bp}$. Peaks were then called by MACS by default parameters, using IgG as control ${ }^{67}$. Histone modification level was defined as non-duplicate reads in peaks (PR) normalized by total non-duplicate reads (NR), $D=P R / N R$. Modification level variation $\left(\right.$ PTEN $^{-1-}$ versus PTEN $\left.{ }^{+/+}>2\right)$ was assessed by the following command: macs2 bdgdiff $-\mathrm{t} 1 \mathrm{k} 4 \mathrm{wt} . \mathrm{bdg}-\mathrm{t} 2 \mathrm{k} 4 \mathrm{ko} . \mathrm{bdg}-\mathrm{cl}$ k4wt_control_lambda.bdg -c2 k4ko_control_lambda.bdg -d1 6191314 -d2 6191314 -outdir./ - C 0.3 -g 200 -l 50

CREB-binding sites analysis. Promoter for CREB-binding sites analysis was defined as $3 \mathrm{~kb}$ upstream of TSS and $300 \mathrm{bp}$ downstream of TSS. CREB-binding sites with $P$-value less than $1 \mathrm{E}-4$ among the promoter regions were found by FIMO ${ }^{68}$ using CREB motif downloaded from MEME database (MEME version 4.4).

Genome-wide CNV analysis. Reads from whole-genome DNA sequencing were mapped to the human reference genome (hg19 from UCSC) by BWA. Signal of $500 \mathrm{~kb}$ window $\mathrm{CNV}$ analysis was calculated by the following formula, $C=5^{*} d^{*} 10^{9} /\left((500,000-t)^{*} b\right)$, in which $d$ is the base number in $500 \mathrm{~kb}$ that mapped to the reference, $t$ is the number of $\mathrm{N}$ in the $500 \mathrm{~kb}$ window and $b$ is the total bases that mapped to the reference.

RRBS and TA-RRBS data analysis. First, sequencing reads were trimmed using trim_galore_v0.3.3 by the following command, trim_galore --quality 20 --phred33 --stringency 3 --gzip --length 50 --rrbs --paired --trim1 --output_dir \$out \$raw1 $\$$ raw2. Then trimmed reads were mapped to the hg19 reference genome by Bismark $^{69}$ and Bowtie ${ }^{70}$ using default parameters. Levels of $5 \mathrm{hmC}$ were determined by $\mathrm{C} / \mathrm{C}+\mathrm{T}$ of TA-RRBS and $5 \mathrm{mC}$ were determined by $(\mathrm{C} /(\mathrm{C}+\mathrm{T})-5 \mathrm{hmC} \%)$. In our study, only genome regions with coverage $>=5$ were taken into account.

Statistical analysis. Data were analysed with Student's $t$-tests and were presented as mean \pm standard deviation (s.d. or s.e.m.). $P<0.05$ was considered as significant.

\section{References}

1. Furnari, F. B. et al. Malignant astrocytic glioma: genetics, biology, and paths to treatment. Genes Dev. 21, 2683-2710 (2007).

2. Bianco, R. et al. Loss of PTEN/MMAC1/TEP in EGF receptor-expressing tumor cells counteracts the antitumor action of EGFR tyrosine kinase inhibitors. Oncogene 22, 2812-2822 (2003).

3. Mellinghoff, I. K. et al. Molecular determinants of the response of glioblastomas to EGFR kinase inhibitors. N. Engl. J. Med. 353, 2012-2024 (2005).

4. Nagata, Y. et al. PTEN activation contributes to tumor inhibition by trastuzumab, and loss of PTEN predicts trastuzumab resistance in patients. Cancer cell 6, 117-127 (2004).

5. Dean, M., Fojo, T. \& Bates, S. Tumour stem cells and drug resistance. Nat. Rev. Cancer. 5, 275-284 (2005).

6. Eramo, A. et al. Chemotherapy resistance of glioblastoma stem cells. Cell Death Differ. 13, 1238-1241 (2006).

7. Liu, C. et al. Mosaic analysis with double markers reveals tumor cell of origin in glioma. Cell 146, 209-221 (2011).

8. Visvader, J. E. Cells of origin in cancer. Nature 469, 314-322 (2011).

9. Wang, Y. et al. Expression of mutant p53 proteins implicates a lineage relationship between neural stem cells and malignant astrocytic glioma in a murine model. Cancer Cell 15, 514-526 (2009).

10. Modrek, A. S., Bayin, N. S. \& Placantonakis, D. G. Brain stem cells as the cell of origin in glioma. World J. Stem Cells 6, 43-52 (2014).

11. van der Zee, M. et al. Alterations in Wnt-beta-catenin and Pten signalling play distinct roles in endometrial cancer initiation and progression. J. Pathol. 230, 48-58 (2013).

12. Alcantara Llaguno, S. et al. Malignant astrocytomas originate from neural stem/progenitor cells in a somatic tumor suppressor mouse model. Cancer Cell 15, 45-56 (2009).

13. Zheng, H. et al. p53 and Pten control neural and glioma stem/progenitor cell renewal and differentiation. Nature 455, 1129-1133 (2008).

14. Chen, J., McKay, R. M. \& Parada, L. F. Malignant glioma: lessons from genomics, mouse models, and stem cells. Cell 149, 36-47 (2012).

15. Rangarajan, A. \& Weinberg, R. A. Opinion: Comparative biology of mouse versus human cells: modelling human cancer in mice. Nat. Rev. Cancer 3, 952-959 (2003)

16. Wright, W. E. \& Shay, J. W. Telomere dynamics in cancer progression and prevention: fundamental differences in human and mouse telomere biology. Nature Med. 6, 849-851 (2000).

17. Grskovic, M., Javaherian, A., Strulovici, B. \& Daley, G. Q. Induced pluripotent stem cells--opportunities for disease modelling and drug discovery. Nat. Rev. Drug Discov. 10, 915-929 (2011).
18. Soldner, F. \& Jaenisch, R. Medicine. iPSC disease modeling. Science 338, 1155-1156 (2012).

19. Liu, G. H. et al. Recapitulation of premature ageing with iPSCs from Hutchinson-Gilford progeria syndrome. Nature 472, 221-225 (2011).

20. Liu, G. H. et al. Progressive degeneration of human neural stem cells caused by pathogenic LRRK2. Nature 491, 603-607 (2012).

21. Liu, G. H., Ding, Z. \& Izpisua Belmonte, J. C. iPSC technology to study human aging and aging-related disorders. Curr. Opin. Cell Biol. 24, 765-774 (2012).

22. Liu, G. H. et al. Modelling Fanconi anemia pathogenesis and therapeutics using integration-free patient-derived iPSCs. Nat. Commun. 5, 4330 (2014).

23. Zhang, W. et al. A Werner syndrome stem cell model unveils heterochromatin alterations as a driver of human aging. Science 348, 1160-1163 (2015).

24. Zuckermann, M. et al. Somatic CRISPR/Cas9-mediated tumour suppressor disruption enables versatile brain tumour modelling. Nat. Commun. 6, 7391 (2015).

25. Drost, J. et al. Sequential cancer mutations in cultured human intestinal stem cells. Nature 521, 43-U329 (2015).

26. Matano, M. et al. Modeling colorectal cancer using CRISPR-Cas9-mediated engineering of human intestinal organoids. Nature Med. 21, 256-262 (2015).

27. Shen, W. H. et al. Essential role for nuclear PTEN in maintaining chromosomal integrity. Cell 128, 157-170 (2007).

28. Weng, L., Brown, J. \& Eng, C. PTEN induces apoptosis and cell cycle arrest through phosphoinositol-3-kinase/Akt-dependent and -independent pathways. Hum. Mol. Genet. 10, 237-242 (2001).

29. Zhao, H., Dupont, J., Yakar, S., Karas, M. \& LeRoith, D. PTEN inhibits cell proliferation and induces apoptosis by downregulating cell surface IGF-IR expression in prostate cancer cells. Oncogene 23, 786-794 (2004).

30. Pollard, S. M. et al. Glioma stem cell lines expanded in adherent culture have tumor-specific phenotypes and are suitable for chemical and genetic screens. Cell stem cell 4, 568-580 (2009).

31. Suva, M. L. et al. Reconstructing and reprogramming the tumor-propagating potential of glioblastoma stem-like cells. Cell 157, 580-594 (2014).

32. Louis, D. N. et al. The 2007 WHO classification of tumours of the central nervous system. Acta Neuropathol. 114, 97-109 (2007).

33. Zhu, Z. et al. Targeting self-renewal in high-grade brain tumors leads to loss of brain tumor stem cells and prolonged survival. Cell Stem Cell 15, 185-198 (2014).

34. Cheng, L. et al. Glioblastoma stem cells generate vascular pericytes to support vessel function and tumor growth. Cell 153, 139-152 (2013).

35. Singh, S. K. et al. Identification of human brain tumour initiating cells. Nature 432, 396-401 (2004).

36. Bao, S. et al. Glioma stem cells promote radioresistance by preferential activation of the DNA damage response. Nature 444, 756-760 (2006).

37. Kawamura, T. et al. Linking the p53 tumour suppressor pathway to somatic cell reprogramming. Nature 460, 1140-U1107 (2009).

38. Chin, Y. R., Yuan, X., Balk, S. P. \& Toker, A. PTEN-deficient tumors depend on AKT2 for maintenance and survival. Cancer Discov. 4, 942-955 (2014).

39. Chow, L. M. \& Baker, S. J. PTEN function in normal and neoplastic growth. Cancer Lett. 241, 184-196 (2006).

40. Saga, I. et al. Integrated analysis identifies different metabolic signatures for tumor-initiating cells in a murine glioblastoma model. Neuro Oncol. 16, 1048-1056 (2014).

41. Vander Heiden, M. G., Cantley, L. C. \& Thompson, C. B. Understanding the Warburg effect: the metabolic requirements of cell proliferation. Science 324, 1029-1033 (2009).

42. Chiappalupi, S., Riuzzi, F., Fulle, S., Donato, R. \& Sorci, G. Defective RAGE activity in embryonal rhabdomyosarcoma cells results in high PAX7 levels that sustain migration and invasiveness. Carcinogenesis 35, 2382-2392 (2014).

43. Riuzzi, F. et al. RAGE signaling deficiency in rhabdomyosarcoma cells causes upregulation of PAX7 and uncontrolled proliferation. J. Cell Sci. 127, 1699-1711 (2014).

44. Chen, Z. H. et al. PTEN interacts with histone $\mathrm{H} 1$ and controls chromatin condensation. Cell Rep. 8, 2003-2014 (2014).

45. Gu, T. T. et al. CREB is a novel nuclear target of PTEN phosphatase. Cancer Res. 71, 2821-2825 (2011).

46. Ikenoue, T., Inoki, K., Zhao, B. \& Guan, K. L. PTEN acetylation modulates its interaction with PDZ domain. Cancer Res. 68, 6908-6912 (2008).

47. Gunther, H. S. et al. Glioblastoma-derived stem cell-enriched cultures form distinct subgroups according to molecular and phenotypic criteria. Oncogene 27, 2897-2909 (2008).

48. Filbin, M. G. et al. Coordinate activation of Shh and PI3K signaling in PTEN-deficient glioblastoma: new therapeutic opportunities. Nature Med. 19, 1518-1523 (2013).

49. Deans, A. J. \& West, S. C. DNA interstrand crosslink repair and cancer. Nat. Rev. Cancer 11, 467-480 (2011). 
50. Amiri, A. et al. Pten deletion in adult hippocampal neural stem/progenitor cells causes cellular abnormalities and alters neurogenesis. J. Neurosci 32, 5880-5890 (2012).

51. Groszer, M. et al. PTEN negatively regulates neural stem cell self-renewal by modulating G0-G1 cell cycle entry. Proc. Natl Acad. Sci. USA 103, 111-116 (2006).

52. Chin, L. et al. Comprehensive genomic characterization defines human glioblastoma genes and core pathways. Nature 455, 1061-1068 (2008).

53. Muller, F. J., Snyder, E. Y. \& Loring, J. F. Gene therapy: can neural stem cells deliver? Nat. Rev. Neurosci. 7, 75-84 (2006).

54. Liu, G. H. et al. Targeted gene correction of laminopathy-associated LMNA mutations in patient-specific iPSCs. Cell Stem Cell 8, 688-694 (2011).

55. Azari, H. et al. Isolation and expansion of human glioblastoma multiforme tumor cells using the neurosphere assay. J. Vis. Exp. 56, e3633 (2011).

56 . $\mathrm{Yu}$, J. et al. Induced pluripotent stem cell lines derived from human somatic cells. Science 318, 1917-1920 (2007).

57. Lensch, M. W., Schlaeger, T. M., Zon, L. I. \& Daley, G. Q. Teratoma formation assays with human embryonic stem cells: a rationale for one type of humananimal chimera. Cell Stem Cell 1, 253-258 (2007).

58. Chiu, Y. W. et al. Baicalein inhibits the migration and invasive properties of human hepatoma cells. Toxicol. Appl. Pharmacol. 255, 316-326 (2011).

59. Reitman, Z. J. et al. Profiling the effects of isocitrate dehydrogenase 1 and 2 mutations on the cellular metabolome. Proc. Natl Acad. Sci. USA 108, 3270-3275 (2011).

60. Dahl, J. A. \& Collas, P. A rapid micro chromatin immunoprecipitation assay (microChIP). Nat. Protoc. 3, 1032-1045 (2008).

61. Wen, L. et al. Whole-genome analysis of 5-hydroxymethylcytosine and 5methylcytosine at base resolution in the human brain. Genome Biol. 15, R49 (2014).

62. Trapnell, C., Pachter, L. \& Salzberg, S. L. TopHat: discovering splice junctions with RNA-Seq. Bioinformatics 25, 1105-1111 (2009).

63. Roberts, A., Pimentel, H., Trapnell, C. \& Pachter, L. Identification of novel transcripts in annotated genomes using RNA-Seq. Bioinformatics 27, 2325-2329 (2011).

64. Trapnell, C. et al. Differential gene and transcript expression analysis of RNAseq experiments with TopHat and Cufflinks. Nat. Protoc. 7, 562-578 (2012).

65. Huang, da, W., Sherman, B. T. \& Lempicki, R. A. Bioinformatics enrichment tools: paths toward the comprehensive functional analysis of large gene lists. Nucleic Acids Res. 37, 1-13 (2009).

66. Li, H. \& Durbin, R. Fast and accurate long-read alignment with BurrowsWheeler transform. Bioinformatics 26, 589-595 (2010).

67. Zhang, Y. et al. Model-based analysis of ChIP-Seq (MACS). Genome Biol. 9, R137 (2008).

68. Grant, C. E., Bailey, T. L. \& Noble, W. S. FIMO: scanning for occurrences of a given motif. Bioinformatics 27, 1017-1018 (2011).

69. Krueger, F. \& Andrews, S. R. Bismark: a flexible aligner and methylation caller for Bisulfite-Seq applications. Bioinformatics 27, 1571-1572 (2011).

70. Langmead, B., Trapnell, C., Pop, M. \& Salzberg, S. L. Ultrafast and memoryefficient alignment of short DNA sequences to the human genome. Genome Biol. 10, R25 (2009)

\section{Acknowledgements}

We are grateful to Jianfeng Lei from the Capital Medical University and Yihui Xu from Institute of Biophysics for technical assistance, and Yang Zhao for administrative assistance. This work was supported by the National Basic Research Program of China
(973 Program, 2015CB964800; 2014CB910503; 2014CB964600; 2012CB966704; 2011CB504100), the Strategic Priority Research Program of the Chinese Academy of Sciences (XDA01020312), the Beijing Natural Science Foundation $(7141005 ; 5142016)$, the National High Technology Research and Development Program of China (2015AA020307), the National Natural Science Foundation of China (NSFC: 81330008 ; 31222039; 31201111; 81371342; 81300261; 81300677; 81271266; 81471414; 81422017; 81401159; 31322039), the Program of Beijing Municipal Science and Technology Commission (Z151100003915072), the Key Research Program of the Chinese Academy of Sciences (KJZDEW-TZ-L05), the Thousand Young Talents program of China, National Laboratory of Biomacromolecules (012kf02;2013kf05;2013kf11;2014kf02;2015kf10), the State Key Laboratory of Drug Research (SIMM1302KF-17) and the China Postdoctoral Science Foundation Grant (2013M530751). J.C.I.B. was supported by the Salk Institute Cancer Center Support Grant, Universidad Católica San Antonio de Murcia (UCAM), the G. Harold and Leila Y. Mathers Charitable Foundation and the Leona M. and Harry B. Helmsley Charitable Trust (PG-2012-MED002). W.Z. was supported by NIH grants K99/ R00 CA158055, the V Scholar award, American Cancer Society seed grant, Breast Cancer Research Award and Oberley Award (National Cancer Institute Award P30CA086862) from Holden Comprehensive Cancer Center at the University of Iowa, and startup funds from the Department of Pathology, University of Iowa.

\section{Author contributions}

S.D., G.Y. and X.L. performed the majority of the experiments. F.T., X.X., X.L. and J. L. carried out the metabolomics, genomic and epigenomic analyses. S.D., G.Y., X.X., J.Q., L.F., C.R.E., J.Y. and K.S. generated iPSC, ESC/iPSC characterization and gene editing. S.D., G.Y. and J.Q. performed cell culture and differentiation. R.R. S.D., G.Y. and Y.L. performed animal experiments. S.D., G.Y. and R.B. X.W., T.J., C.Z. and Z.C. performed sample collection and data analyses. J.C.I.B., J.W., Weizhou Zhang, F.Y., H.G. and Weiqi Zhang performed data analysis and wrote the manuscript. S. D., G.Y., X.L., J.Q., F.T. and G.-H.L. conceived this study and wrote the manuscript.

\section{Additional information}

Accession codes: The RNA-seq, ChIP-seq, RRBS, TA-RRBS sequencing data sets have been deposited in NCBI Gene Expression Omnibus (GEO) under accession code GSE61794. And the whole-genome DNA sequencing data for CNV analysis are deposited to the Sequence Read Archive (SRA) under accession number of SRP048488.

Supplementary Information accompanies this paper at http://www.nature.com/ naturecommunications

Competing financial interests: The authors declare no competing financial interests.

Reprints and permission information is available online at http://npg.nature.com/ reprintsandpermissions/

How to cite this article: Duan, S. et al. PTEN deficiency reprogrammes human neural stem cells towards a glioblastoma stem cell-like phenotype. Nat. Commun. 6:10068 doi: $10.1038 /$ ncomms10068 (2015)

This work is licensed under a Creative Commons Attribution 4.0 International License. The images or other third party material in this article are included in the article's Creative Commons license, unless indicated otherwise in the credit line; if the material is not included under the Creative Commons license, users will need to obtain permission from the license holder to reproduce the material. To view a copy of this license, visit http://creativecommons.org/licenses/by/4.0/ 